\title{
A Numerical Study on the Performance of Nonlinear Models of a Microvibration Isolator
}

\author{
Jie Wang, Shougen Zhao, and Dafang Wu \\ School of Aeronautic Science and Engineering, Beijing University of Aeronautics and Astronautics, Beijing 100191, China
}

Correspondence should be addressed to Shougen Zhao; zshougen@buaa.edu.cn

Received 26 February 2014; Accepted 27 May 2014; Published 22 July 2014

Academic Editor: Chao Tao

Copyright (C) 2014 Jie Wang et al. This is an open access article distributed under the Creative Commons Attribution License, which permits unrestricted use, distribution, and reproduction in any medium, provided the original work is properly cited.

A non-Newton fluid microvibration isolator is studied in this paper and several nonlinear models are firstly presented to characterize its vibration behaviors due to the complicated effects of internal structure, external excitation, and fluid property. On the basis of testing hysteretic loops, the generalized pattern search (GPS) algorithm of MATLAB optimization toolbox is used to identify the model parameters. With the use of the fourth-order Runge-Kutta method, the performance of these nonlinear models is further estimated. The results show that, in the cases of force excitation (FE), the generalized nonlinear model (GNM) and the complicated model (CM) can properly characterize the physical vibration in the frequency band of 5-20 Hz. However, in the frequency band of 30-200 Hz, the Maxwell model shows more excellent performance. After the application of orthogonal testing method, several important factors, for example, damping coefficient and flow index, are obtained; then a parametric analysis is carried out with the purpose of further studying the influences of nonlinear model parameters. It can be seen that only the GNM and $\mathrm{CM}$ can consider the above nonlinear effects in both the FE cases and the foundation displacement excitation (FDE) cases, but the $\mathrm{CM}$ is not convenient to use in practice.

\section{Introduction}

In recent years, the international space technology has made a rapid development, which means that the categories, functions, and structural format of spacecraft are becoming increasingly various and complex [1]. In particular, the high resolution remote sensing satellites are one of the research hotspots of high precision spacecraft; for example, the American KH-13 surveillance satellite achieves an observational resolution of 0.05 meter, and the commercial remote sensing satellite GeoEye-1 launched in September 2008 acquires a resolution of 0.41 meter [2]. Due to the effects of bearing disturbance, static and dynamic imbalance of momentum/reaction wheels, the high frequency jitter may propagate to spacecraft body structure and then to optical payload; thus the image quality and the resolution performance will probably be affected seriously [3]. In order to satisfy the strict line-of-sight performance and stability requirements of high precision spacecraft, the amplitude of vibration should be reduced to the order of micrometers or even nanometers [4], and a commonly used method to solve this dilemma is inserting oil microvibration isolators between the jitter source and spacecraft or/and between the optical payload and spacecraft [5]. Since a reasonable vibration model is the basis to accurately characterize the vibration isolation performance [6], Davis et al. [7] and Anderson et al. [8] constructed a linear model for a damper named D-Strut, and its vibration isolation performance was theoretically analyzed and experimentally validated. However, most of viscous fluid isolators contain non-Newton fluid, and the flow states, for example, laminar and turbulent, are closely related to the frequency of flow oscillation. Moreover, nonlinear damping such as coulomb friction also exists in the piston damper [5], so the vibration behaviors can only be completely described by the nonlinear models. Ibrahim [9] presented a comprehensive review of nonlinear passive vibration isolation and gave a patulous introduction of several nonlinear vibration isolators. With the use of output frequency response function (OFRF), Lang et al. [10] investigated the effects of cubic nonlinear viscous damping on the vibration isolation performance of a single degree of freedom (DOF) system, and the results show that only the transmissibility at resonant region can 
be affected, while the transmissibility at nonresonant region is almost the same as that of a linear case. Peng et al. [11] expanded the investigation into a multi-DOF structure and obtained similar results. Tang and Brennan [12] studied a kind of nonlinear horizontal damping and compared its force and displacement transmissibility with that of cubic damping, respectively. Moreover, Ping [13] proposed a nonlinear model which contains nonlinear stiffness and various nonlinear dampings for a kind of gas-and-oil-mixed shock absorber, and the influences of each factor on the performance of resisting violent impact and attenuating vibration were thoroughly analyzed. Besides, a gauze-fluid damping shock absorber was examined later, whose inner coupling damping force and nonlinear stiffness were also theoretically analyzed, experimentally tested, and numerically simulated [14]. Chandra Shekhar et al. [15] numerically studied four kinds of nonlinear damping strategies, that is, an isolator with a coulomb damper, a three-parameter isolator, an isolator appended by an absorber, and a two-stage isolator, to improve the performance of nonlinear shock isolators. Besides, a shock isolator with cubic nonlinear damping and stiffness was also investigated and the closed form solution was obtained by a combined method of straightforward perturbation and Laplace transformation; thus it is convenient to obtain analytical solution at any time and there is no need to integrate from the start point [16]. Narkhede and Sinha [17] studied a kind of shock absorber which installs an accumulator housing next to the fluid reservoir, so the fluid elastic effect which is similar to that of a compressed balloon is vanished; thus the damping force is proportional to the fractional power-law of velocity. Lu et al. $[18,19]$ presented a kind of long-stroke fluid damper of seismic engineering; the proposed mathematical model which is called generalized Maxwell model (GMM) mainly contains four parameters, that is, stiffness coefficient, damping coefficient, stiffness exponent, and damping exponent, and it can accurately simulate/characterize the hysteretic behaviors of the damper. Yang et al. [20] analyzed the dynamic and power flow behaviors of a nonlinear vibration model, whose establishment is based on a kind of negative stiffness mechanism.

An ideal microvibration isolator should survive from the launch stage to protect the payload, at which the FDE amplitude is large and the nonlinear effect of fluid is very strong. Besides, it also should keep perfect working state in orbit to improve the image quality, in which the FE amplitude is small and the vibration displacement is on the order of micrometers or even nanometers. Thus the vibration isolation performance at different stages and the corresponding key factors should be seriously considered. Peng et al. [21] used harmonic balance method to investigate the effects of cubic nonlinear damping on the performance of a passive vibration isolation system and concluded that linear and nonlinear damping have distinct influences on the absolute displacement transmissibility, relative displacement transmissibility, and force transmissibility. Based on the combination of Fourier expansion and harmonic balance method, Ravindra and Mallik [22] obtained the first-order approximated solution of a single DOF model whose $p$ th power damping and $q$ th power stiffness are placed in parallel and estimated the corresponding force and displacement transmissibility. Laalej et al. [23] verified the effects of cubic nonlinear damping by an experiment, in which the vertical force transmissibility of Stewart or Hexapod platform was considered.

Based on the above review, vibration isolators always behave nonlinearly due to the complex effects of internal structure, external excitation, and fluid property; and the hypotheses, for example, the compressibility or incompressibility of viscous fluid, are also always made to simplify the modeling process. Besides, it is realized that a comprehensive study on various nonlinear models of a microvibration isolator is needed to provide a proper basis for engineering applications. Thus, several nonlinear models are firstly constructed to characterize the vibration behaviors, and the performance of them is estimated based on a comparison of hysteretic loops between simulation and test. Then a parametric analysis of several important factors is executed to further study their influences on the vibration isolation performance.

\section{Vibration Modeling and Analysis}

Figure 1 shows the structure schematic of a microvibration isolator; the left and right connecting end faces are connected to the base and isolated mass, respectively. The stiffness coefficients of the outer tube, inner tube, and the crust of fluid reservoir are $k_{1}, k_{2}$, and $k_{3}$, respectively. The damping component is made up of the fluid reservoir, bellows, and the damping orifice. When the isolator is excited by an external force and the axial elastic deformation happens, the fluid of reservoir is forced to flow through the damping orifice; thus the damping force of isolator is generated, which mainly comes from the shearing effect of fluid in the damping orifice.

As non-Newton fluid silicon oil is contained in the microvibration isolator, and the flow state is assumed as laminar flow; thus the shear stress $\tau_{w}$ of fluid in the damping orifice can be expressed as

$$
\tau_{w}=\frac{\Delta P R}{2 L}
$$

where $\Delta P, R$, and $L$ are the pressure difference, radius, and length of the damping orifice, respectively. The average flow velocity $v$ of the damping orifice is

$$
v=\left(\frac{\Delta P}{2 k L}\right)^{1 / n} \frac{n}{1+3 n} R^{(n+1) / n},
$$

where $k$ is the consistency coefficient and $n$ is the flow index. Equation (2) can be rewritten as

$$
\Delta P=\left(\frac{1+3 n}{n} v\right)^{n} 2 k L R^{-(n+1)} .
$$

With the hypothesis of incompressible fluid, the continuity condition of fluid can be expressed as

$$
v \frac{\pi d^{2}}{4}=V \frac{\pi D^{2}}{4}
$$




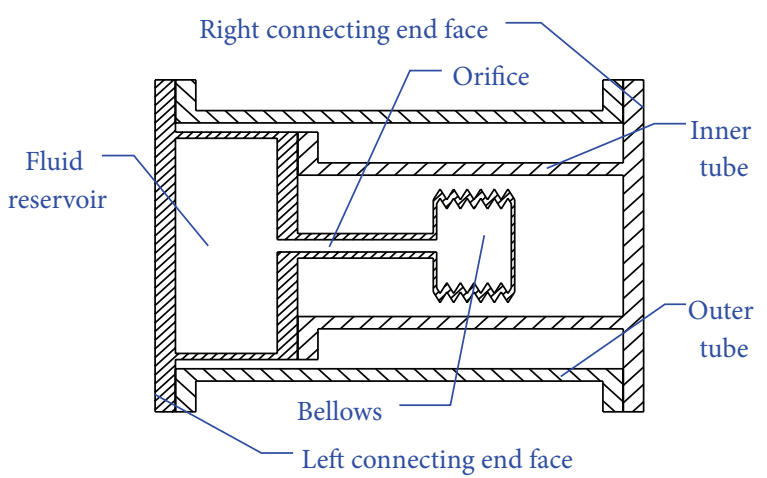

FIGURE 1: The schematic diagram of a microvibration isolator.

where $d$ and $D$ are the diameters of the damping orifice and fluid reservoir, respectively, and $V$ is the relative velocity between the two ends of fluid reservoir.

After combining (1)-(4), the damping force $P_{D}$ can be written as

$$
P_{D}=\left(\frac{1+3 n}{n}\right)^{n} D^{2 n} \pi k L 2^{n} d^{-3 n+1} V^{n},
$$

so the damping force $P_{D}$ is proportional to the $n$th power of velocity $V$. Since the compressibility exists in real fluid, thus the damping force should be in series with the volumetric stiffness of fluid. With an integrated consideration of compressibility, incompressibility, nonlinear damping, and nonlinear stiffness, this paper firstly presents a complicated model as illustrated in Figure 2. The damping coefficient $c_{1}$ and the stiffness coefficient $k_{4}$ are placed in series, and the damping coefficient $c_{2}$ is placed in parallel with them. Besides, the corresponding damping exponents and stiffness exponent are $n_{1}, n_{2}$, and $n_{k}$, respectively.

The signum function is defined as

$$
\operatorname{sgn}(x)= \begin{cases}1 & \text { if } x>0 \\ 0 & \text { if } x=0 \\ -1 & \text { if } x<0\end{cases}
$$

Accordingly, the equation of motion of this system is

$$
\begin{aligned}
& M \ddot{x}_{p}+k_{1}\left(x_{p}-x_{b}\right)+k_{2}\left(x_{p}-x\right)=F(t) \\
& k_{2}\left(x_{p}-x\right)=k_{3}\left(x-x_{b}\right)+\operatorname{sgn}\left(x-x_{d}\right) k_{4}\left|x-x_{d}\right|^{n_{k}} \\
& \quad+\operatorname{sgn}\left(\dot{x}-\dot{x}_{b}\right) c_{2}\left|\dot{x}-\dot{x}_{b}\right|^{n_{2}} \\
& \operatorname{sgn}\left(x-x_{d}\right) k_{4}\left|x-x_{d}\right|^{n_{k}}=\operatorname{sgn}\left(\dot{x}_{d}-\dot{x}_{b}\right) c_{1}\left|\dot{x}_{d}-\dot{x}_{b}\right|^{n_{1}} .
\end{aligned}
$$

In the cases of FDE, $F(t)=0, x_{b}=A_{1} \cos \omega t$. By setting

$$
\omega_{0}=\sqrt{\frac{k_{1}}{M}}, \quad \tau=\omega_{0} t, \quad \delta_{1}(\tau)=\frac{\left(x_{p}-x_{b}\right)}{A_{1}},
$$

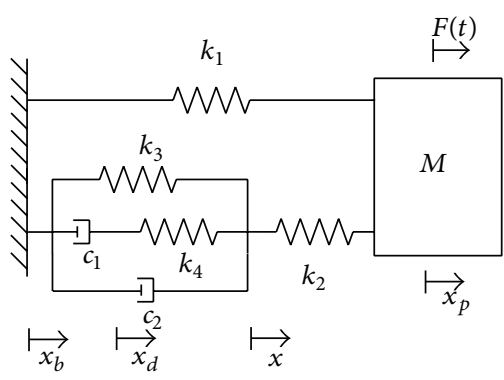

FIgUre 2: Complicated model.

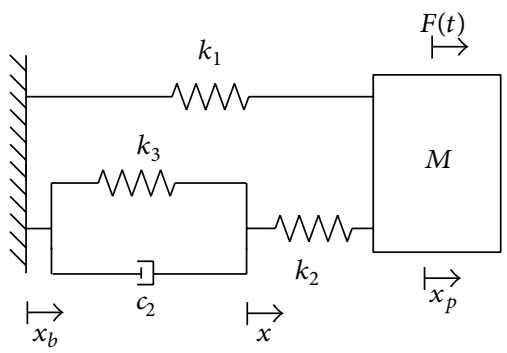

FIgURE 3: Simple model.

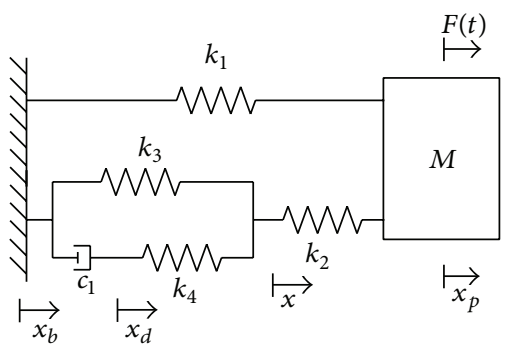

FIGURE 4: Generalized nonlinear model.

$$
\begin{aligned}
& \delta_{2}(\tau)=\frac{\left(x-x_{d}\right)}{A_{1}}, \quad \delta_{3}(\tau)=\frac{\left(x-x_{b}\right)}{A_{1}}, \\
& N_{2}=\frac{k_{2}}{k_{1}}, \quad N_{3}=\frac{k_{3}}{k_{1}}, \quad N_{4}=\frac{k_{4}}{k_{1}}, \quad \lambda=\frac{\omega}{\omega_{0}},
\end{aligned}
$$

and based on the following differential relationship:

$$
\frac{d()}{d t}=\frac{d()}{d \tau} \frac{d \tau}{d t}, \quad \frac{d^{2}()}{d t^{2}}=\frac{d}{d \tau}\left[\frac{d()}{d \tau} \frac{d \tau}{d t}\right] \frac{d \tau}{d t},
$$

(7) can be simplified as

$$
\begin{aligned}
& \delta_{1}(\tau)^{\prime \prime}+\delta_{1}(\tau)+N_{2}\left[\delta_{1}(\tau)-\delta_{3}(\tau)\right]=\lambda^{2} \cos (\lambda \tau), \\
& N_{2}\left[\delta_{1}(\tau)-\delta_{3}(\tau)\right] \\
& =N_{3} \delta_{3}(\tau)+N_{4} A_{1}^{n_{k}-1} \operatorname{sgn}\left(\delta_{2}(\tau)\right)\left|\delta_{2}(\tau)\right|^{n_{k}} \\
& \quad+\varepsilon_{21} \operatorname{sgn}\left(\delta_{3}(\tau)^{\prime}\right)\left|\delta_{3}(\tau)^{\prime}\right|^{n_{2}},
\end{aligned}
$$




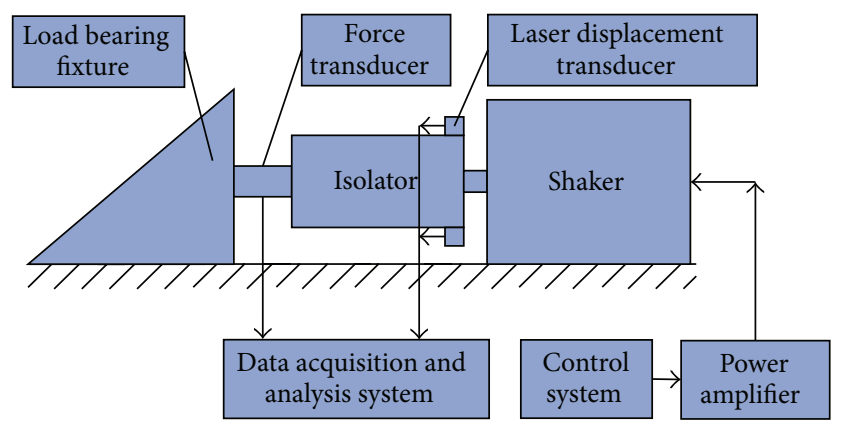

FIGURE 5: The schematic diagram of the test setup of hysteretic loops.

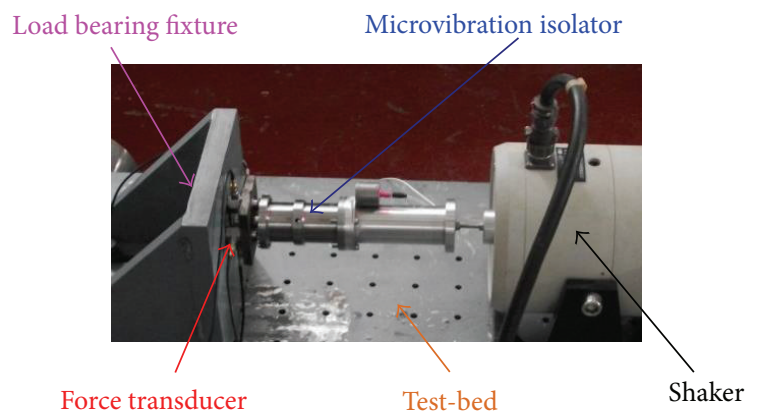

FIgURE 6: The picture of test setup.

$$
\begin{aligned}
N_{4} A_{1}^{n_{k}-1} \operatorname{sgn}\left(\delta_{2}(\tau)\right)\left|\delta_{2}(\tau)\right|^{n_{k}}= & \varepsilon_{11} \operatorname{sgn}\left(\delta_{3}(\tau)^{\prime}-\delta_{2}(\tau)^{\prime}\right) \\
& \times\left|\delta_{3}(\tau)^{\prime}-\delta_{2}(\tau)^{\prime}\right|^{n_{1}},
\end{aligned}
$$

where $\varepsilon_{11}=c_{1} A_{1}^{n_{1}-1} \omega_{0}^{n_{1}-2} / M, \varepsilon_{21}=c_{2} A_{1}^{n_{2}-1} \omega_{0}^{n_{2}-2} / M$.

In the cases of FE, $x_{b}=\dot{x}_{b}=0, F(t)=F_{0} \cos \omega t$. By setting $A_{2}=F_{0} / k_{1}$, (7) can be written as the following form:

$$
\begin{aligned}
M \ddot{x}_{p}+k_{1} x_{p} & +k_{2}\left(x_{p}-x\right)=F(t), \\
k_{2}\left(x_{p}-x\right)= & k_{3} x+k_{4} \operatorname{sgn}\left(x-x_{d}\right)\left|x-x_{d}\right|^{n_{k}} \\
& +c_{2} \operatorname{sgn}(\dot{x})|\dot{x}|^{n_{2}},
\end{aligned}
$$

$$
k_{4} \operatorname{sgn}\left(x-x_{d}\right)\left|x-x_{d}\right|^{n_{k}}=c_{1} \operatorname{sgn}\left(\dot{x}_{d}\right)\left|\dot{x}_{d}\right|^{n_{1}} .
$$

Letting $y_{1}(\tau)=x_{p}(t) / A_{2}, y_{2}(\tau)=\left(x(t)-x_{d}(t)\right) / A_{2}$ and $y_{3}(\tau)=$ $x(t) / A_{2}$, similarly, (11) can be simplified as

$$
\begin{aligned}
& y_{1}(\tau)^{\prime \prime}+y_{1}(\tau)+N_{2}\left[y_{1}(\tau)-y_{3}(\tau)\right]=\cos (\lambda \tau), \\
& N_{2}\left[y_{1}(\tau)-y_{3}(\tau)\right] \\
& =N_{3} y_{3}(\tau)+N_{4} A_{2}^{n_{k}-1} \operatorname{sgn}\left(y_{2}(\tau)\right)\left|y_{2}(\tau)\right|^{n_{k}} \\
& \quad+\varepsilon_{22} \operatorname{sgn}\left(y_{3}(\tau)^{\prime}\right)\left|y_{3}(\tau)^{\prime}\right|^{n_{2}}, \\
& N_{4} A_{2}^{n_{k}-1} \operatorname{sgn}\left(y_{2}(\tau)\right)\left|y_{2}(\tau)\right|^{n_{k}} \\
& \quad=\varepsilon_{12} \operatorname{sgn}\left(y_{3}(\tau)^{\prime}-y_{2}(\tau)^{\prime}\right)\left|y_{3}(\tau)^{\prime}-y_{2}(\tau)^{\prime}\right|^{n_{1}},
\end{aligned}
$$

where $\varepsilon_{12}=c_{1} A_{2}^{n_{1}-1} \omega_{0}^{n_{1}-2} / M, \varepsilon_{22}=c_{2} A_{2}^{n_{2}-1} \omega_{0}^{n_{2}-2} / M$.

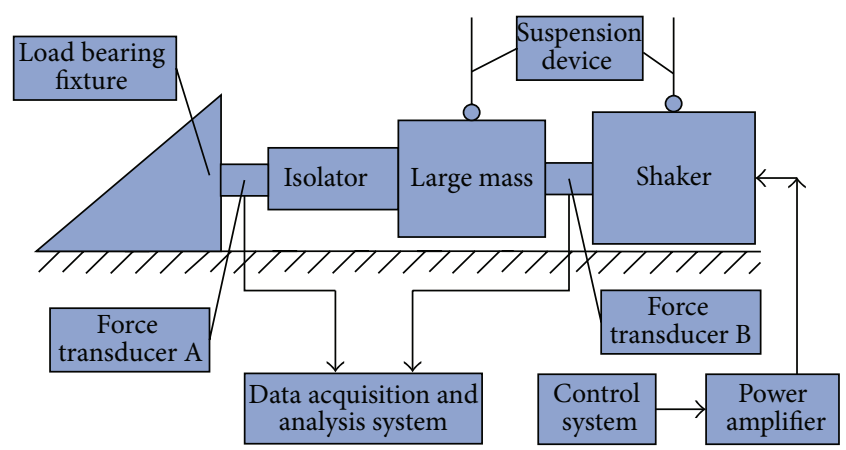

FIGURE 7: The schematic diagram of the test setup of force transmissibility.

Thus, (10) and (12) can be uniformly written as the following nondimensional form:

$$
\begin{aligned}
\Delta_{1}(\tau)^{\prime \prime} & +\Delta_{1}(\tau)+N_{2}\left[\Delta_{1}(\tau)-\Delta_{3}(\tau)\right]=\phi \cos (\lambda \tau), \\
N_{2}\left[\Delta_{1}(\tau)-\Delta_{3}(\tau)\right] & \\
= & N_{3} \Delta_{3}(\tau)+N_{4} A^{n_{k}-1} \operatorname{sgn}\left(\Delta_{2}(\tau)\right)\left|\Delta_{2}(\tau)\right|^{n_{k}} \\
& +\varepsilon_{2} \operatorname{sgn}\left(\Delta_{3}(\tau)^{\prime}\right)\left|\Delta_{3}(\tau)^{\prime}\right|^{n_{2}}, \\
N_{4} A^{n_{k}-1} & \operatorname{sgn}\left(\Delta_{2}(\tau)\right)\left|\Delta_{2}(\tau)\right|^{n_{k}} \\
= & \varepsilon_{1} \operatorname{sgn}\left(\Delta_{3}(\tau)^{\prime}-\Delta_{2}(\tau)^{\prime}\right)\left|\Delta_{3}(\tau)^{\prime}-\Delta_{2}(\tau)^{\prime}\right|^{n_{1}}
\end{aligned}
$$

where $\phi=1$ is for the FE cases and $\phi=\lambda^{2}$ is for the FDE cases. For convenience, these notations, that is, $\Delta_{1}(\tau)=z_{0}(\tau)$, $\Delta_{1}(\tau)^{\prime}=z_{1}(\tau), \Delta_{2}(\tau)=z_{2}(\tau)$, and $\Delta_{3}(\tau)=z_{3}(\tau)$, will be used in the following paragraphs.

2.1. Simple Model. When $c_{1}=0$ or $k_{4}=0, \varepsilon_{1}, \Delta_{2}$, and $z_{2}$ are equal to zero; the CM becomes the following simple model as indicated in Figure 3.

Similarly, (13) can be simplified as

$$
\begin{aligned}
\Delta_{1}(\tau)^{\prime \prime}+\Delta_{1}(\tau)+N_{2} & {\left[\Delta_{1}(\tau)-\Delta_{3}(\tau)\right]=\phi \cos (\lambda \tau), } \\
N_{2}\left[\Delta_{1}(\tau)-\Delta_{3}(\tau)\right]= & N_{3} \Delta_{3}(\tau) \\
& +\varepsilon_{2} \operatorname{sgn}\left(\Delta_{3}(\tau)^{\prime}\right)\left|\Delta_{3}(\tau)^{\prime}\right|^{n_{2}},
\end{aligned}
$$

which has the following form in phase space:

$$
\begin{aligned}
z_{0}^{\prime}= & z_{1}, \\
z_{1}^{\prime}= & -\left(1+N_{2}\right) z_{0}+N_{2} z_{3}+\phi \cos (\lambda \tau), \\
z_{3}^{\prime}= & \operatorname{sgn}\left(\frac{1}{\varepsilon_{2}}\left[N_{2} z_{0}-\left(N_{2}+N_{3}\right) z_{3}\right]\right) \\
& \times\left|\frac{1}{\varepsilon_{2}}\left[N_{2} z_{0}-\left(N_{2}+N_{3}\right) z_{3}\right]\right|^{1 / n_{2}} .
\end{aligned}
$$




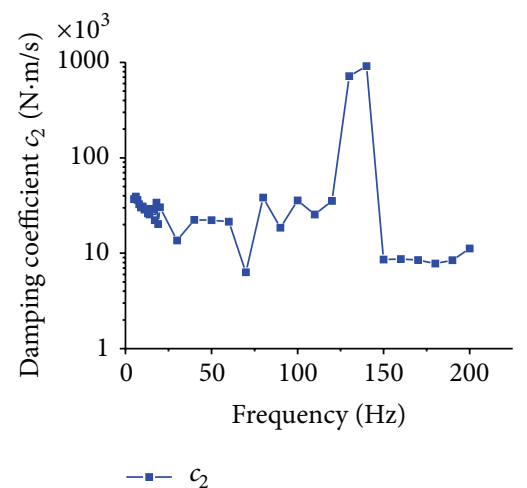

(a)

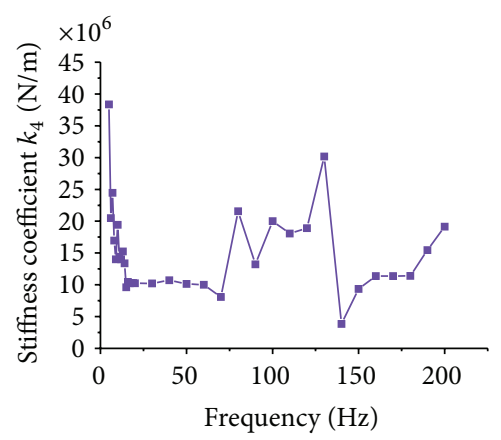

$--k_{4}$

(c)

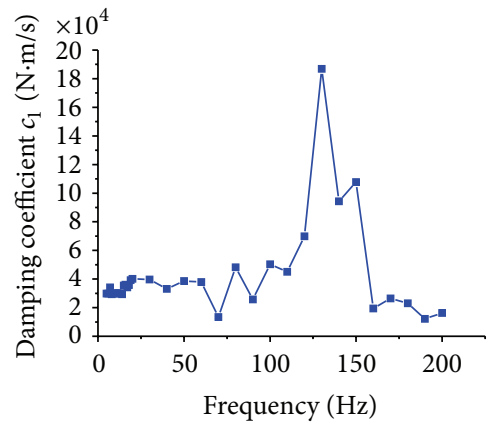

$-\square-c_{1}$

(e)

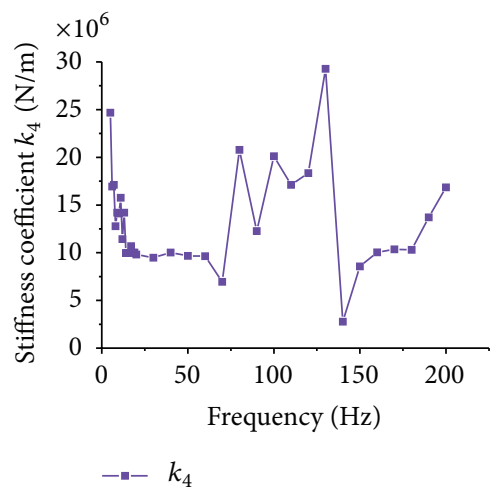

(g)

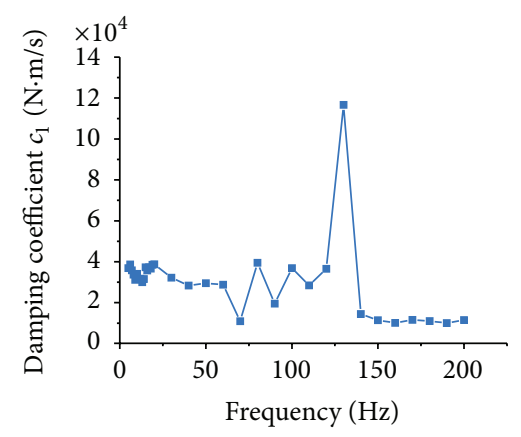

(b)

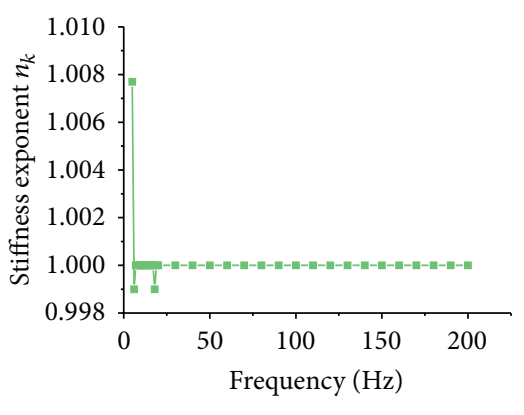

(d)

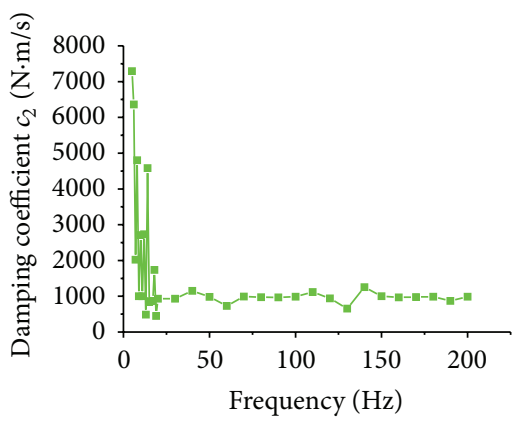

(f)

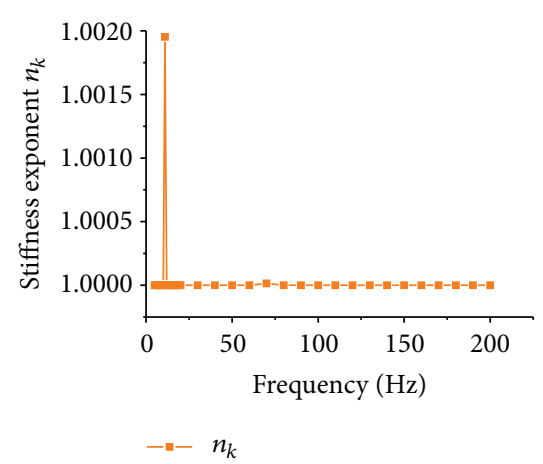

(h)

FIGURE 8: Variations of vibration model parameters with excitation frequency. (a) Simple model; (b), (c), and (d) GNM; (e), (f), (g), and (h) CM. 


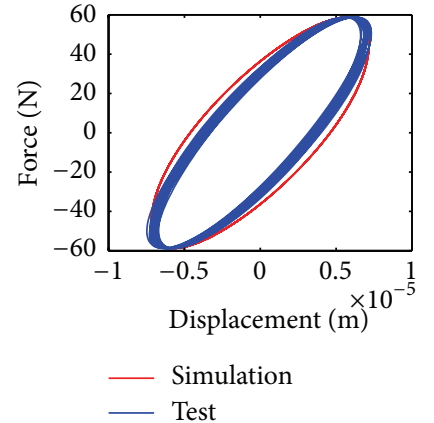

(a1) $f=6 \mathrm{~Hz}$

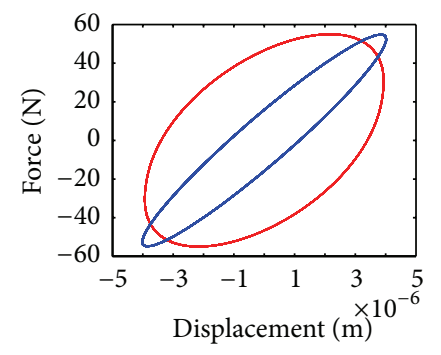

_ Simulation

— Test

(a2) $f=20 \mathrm{~Hz}$

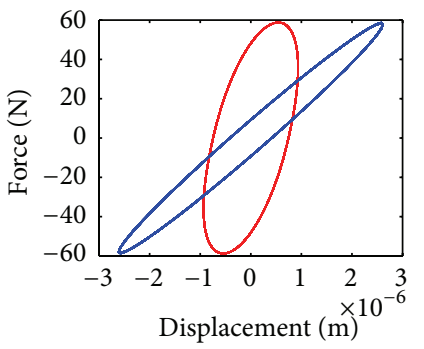

_ Simulation

— Test

(a3) $f=100 \mathrm{~Hz}$

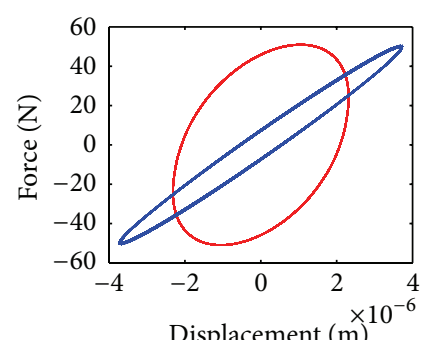

Displacement $(\mathrm{m})$

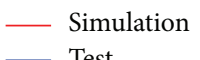

(a4) $f=150 \mathrm{~Hz}$

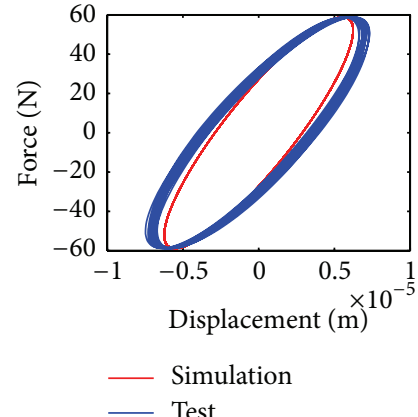

(b1) $f=6 \mathrm{~Hz}$

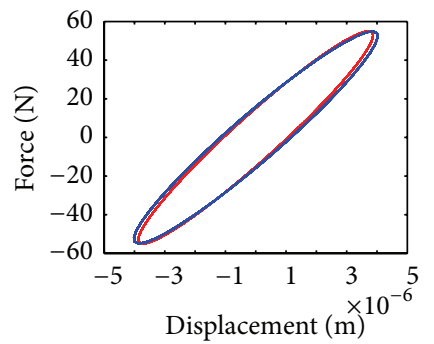

_ Simulation

(b2) $f=20 \mathrm{~Hz}$

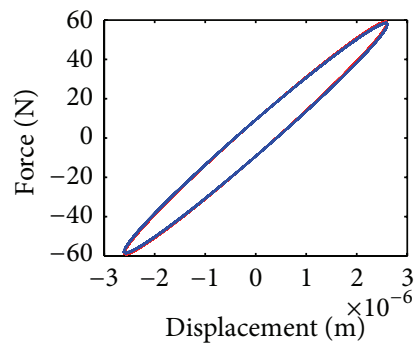

- Simulation

— Test

(b3) $f=100 \mathrm{~Hz}$

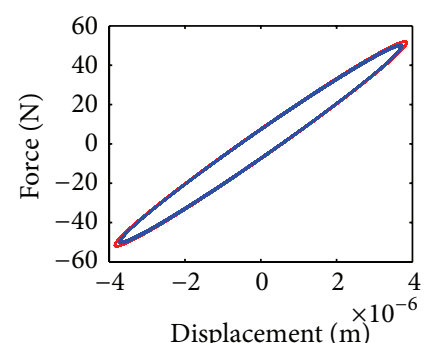

Displacement $(\stackrel{\mathrm{m}}{)}$

_ Simulation

— Test

(b4) $f=150 \mathrm{~Hz}$
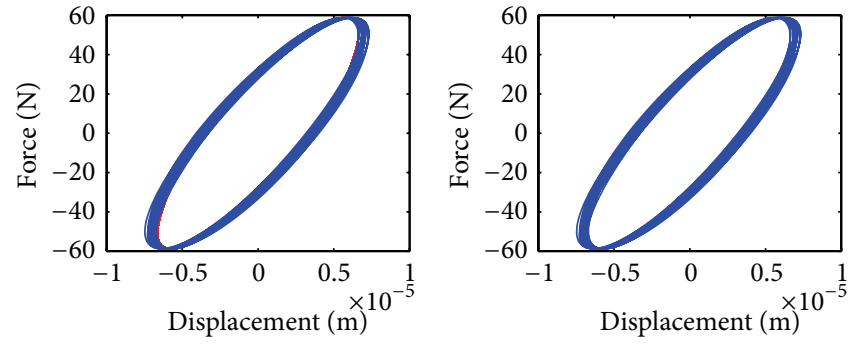

_ Simulation

— Test

(c1) $f=6 \mathrm{~Hz}$

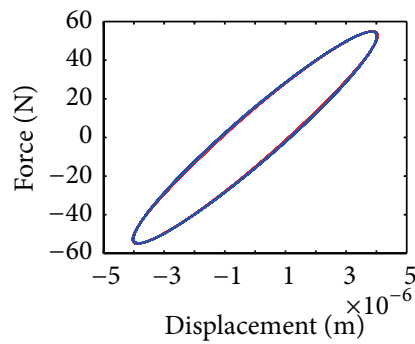

_ Simulation

— Test

(c2) $f=20 \mathrm{~Hz}$

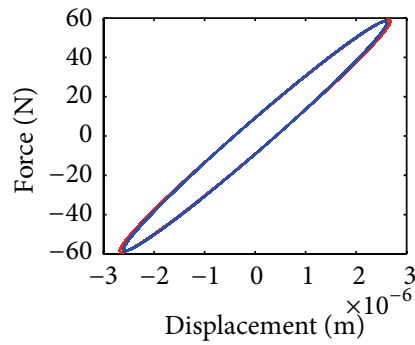

— Simulation

— Test

(c3) $f=100 \mathrm{~Hz}$

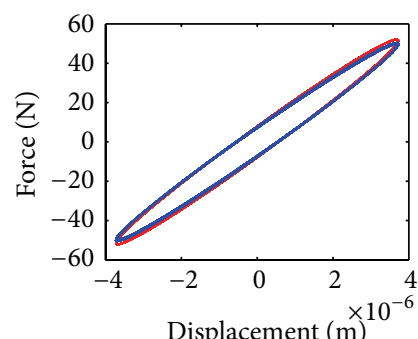

_ Simulation

— Test

(c4) $f=150 \mathrm{~Hz}$
_ Simulation

— Test

(d1) $f=6 \mathrm{~Hz}$

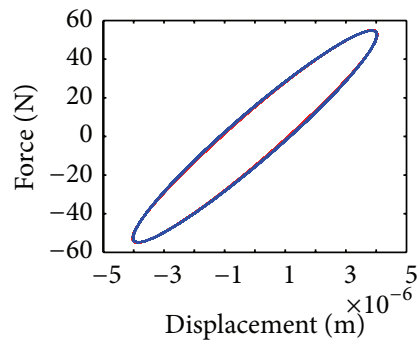

- Simulation

— Test

(d2) $f=20 \mathrm{~Hz}$

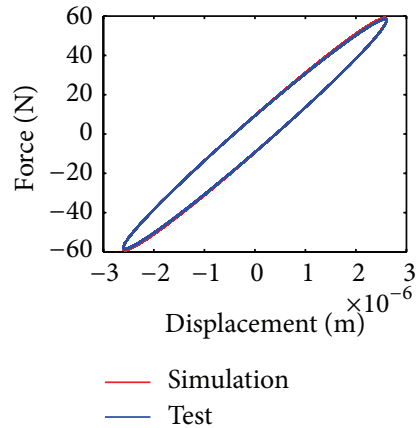

(d3) $f=100 \mathrm{~Hz}$

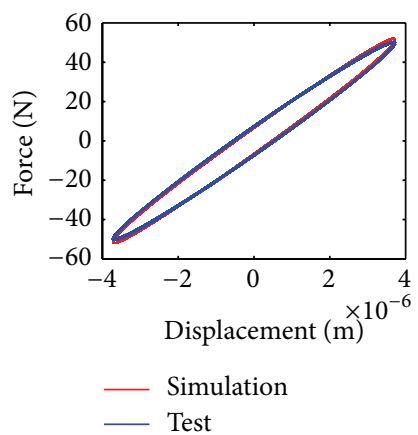

(d4) $f=150 \mathrm{~Hz}$

FIGURE 9: Comparisons of hysteretic loops between simulation and test. (a) Simple model. (b) Linear Maxwell model. (c) GNM. (d) CM. 


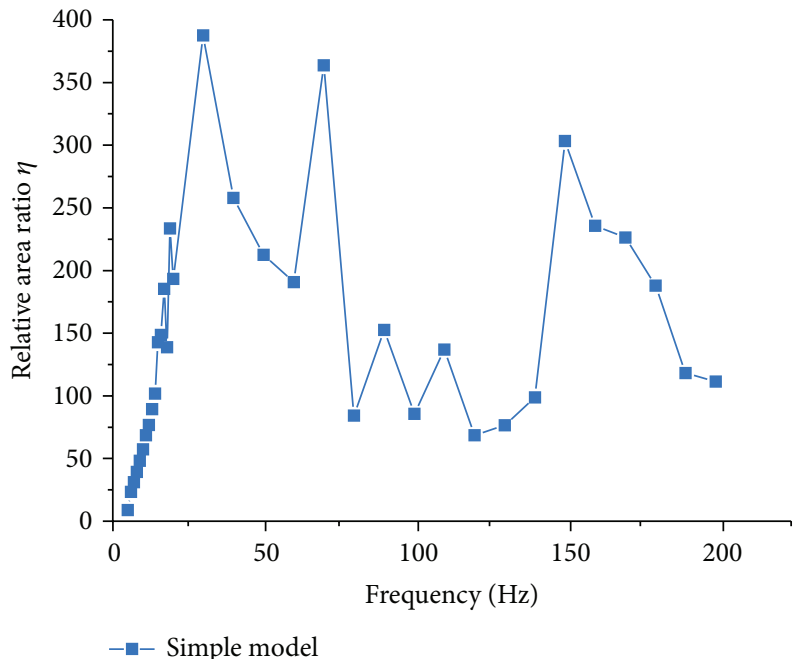

(a)

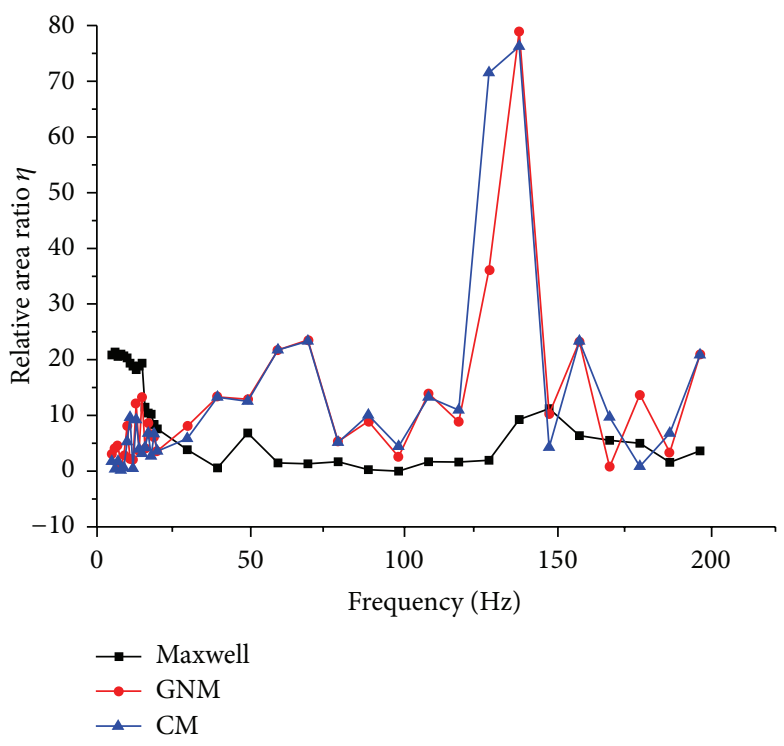

(b)

FIGURE 10: Relative area ratio $\eta$. (a) Simple model; (b) other models.
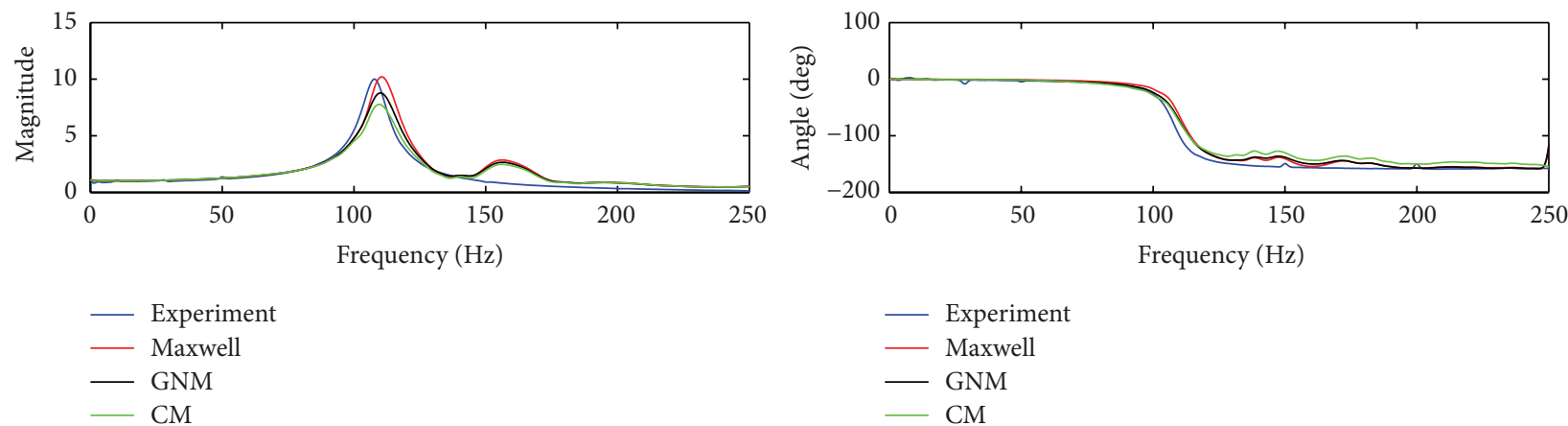

— Experiment

- Maxwell

- GNM

$-\mathrm{CM}$

FIGURE 11: Force transmissibility curves.

If $n_{2}=1$, the system is linear. Letting

$$
\begin{gathered}
\mathbf{Z}_{1}(\tau)=\left(\begin{array}{c}
z_{0} \\
z_{1} \\
z_{3}
\end{array}\right), \\
\mathbf{P}_{3}=\left(\begin{array}{ccc}
0 & 1 & 0 \\
-\left(1+N_{2}\right) & 0 & N_{2} \\
\frac{N_{2}}{\varepsilon_{2}} & 0 & -\frac{N_{2}+N_{3}}{\varepsilon_{2}}
\end{array}\right), \\
\mathbf{T}_{1}(\tau)=\left(\begin{array}{c}
0 \\
\phi \cos (\lambda \tau) \\
0
\end{array}\right),
\end{gathered}
$$

(15) reduces to $\mathbf{Z}_{1}^{\prime}=\mathbf{P}_{1} \mathbf{Z}_{1}+\mathbf{T}_{1}$, whose solution is

$$
\mathbf{Z}_{1}(\tau)=e^{\tau \mathbf{P}_{1}} \mathbf{Z}_{1}(0)+e^{\tau \mathbf{P}_{1}} \int_{0}^{\tau} e^{-u \mathbf{P}_{1}} \mathbf{T}_{1}(u) d u,
$$

where $\varepsilon_{2}=c_{2} / \sqrt{k_{1} M}$.
2.2. Generalized Nonlinear Model. If $c_{2}=0, \varepsilon_{2}$ is equal to zero; the CM reduces to the GNM as shown in Figure 4.

Equation (13) can also be simplified as

$$
\begin{aligned}
& \Delta_{1}(\tau)^{\prime \prime}+\Delta_{1}(\tau)+N_{2}\left[\Delta_{1}(\tau)-\Delta_{3}(\tau)\right]=\phi \cos (\lambda \tau), \\
& N_{2}\left[\Delta_{1}(\tau)-\Delta_{3}(\tau)\right] \\
& \quad=N_{3} \Delta_{3}(\tau)+N_{4} A^{n_{k}-1} \operatorname{sgn}\left(\Delta_{2}(\tau)\right)\left|\Delta_{2}(\tau)\right|^{n_{k}}, \\
& N_{4} A^{n_{k}-1} \operatorname{sgn}\left(\Delta_{2}(\tau)\right)\left|\Delta_{2}(\tau)\right|^{n_{k}} \\
& \quad=\varepsilon_{1} \operatorname{sgn}\left(\Delta_{3}(\tau)^{\prime}-\Delta_{2}(\tau)^{\prime}\right)\left|\Delta_{3}(\tau)^{\prime}-\Delta_{2}(\tau)^{\prime}\right|^{n_{1}} .
\end{aligned}
$$

Letting $\Delta_{3}(\tau)-\Delta_{2}(\tau)=z_{4}(\tau)$, the following first-order ordinary differential equation group can be obtained:

$$
\begin{aligned}
& z_{0}^{\prime}=z_{1} \\
& z_{1}^{\prime}=-\left(1+N_{2}\right) z_{0}+N_{2}\left(z_{2}+z_{4}\right)+\phi \cos (\lambda \tau)
\end{aligned}
$$




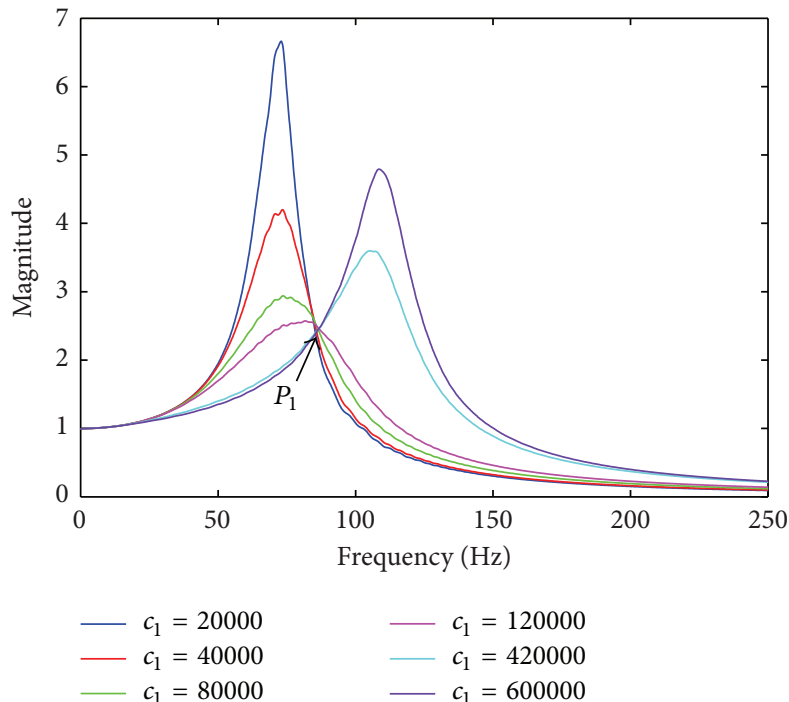

(a)
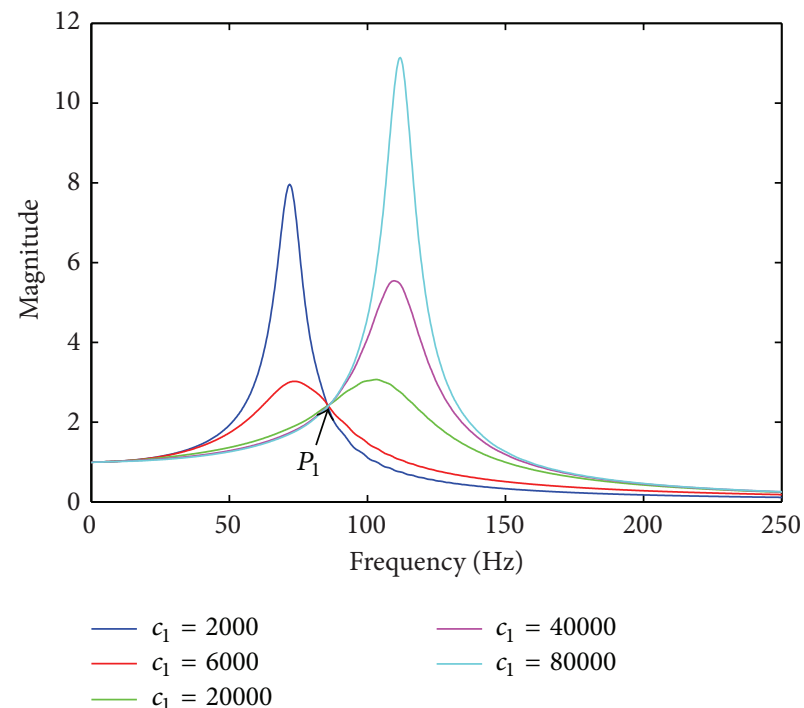

(b)

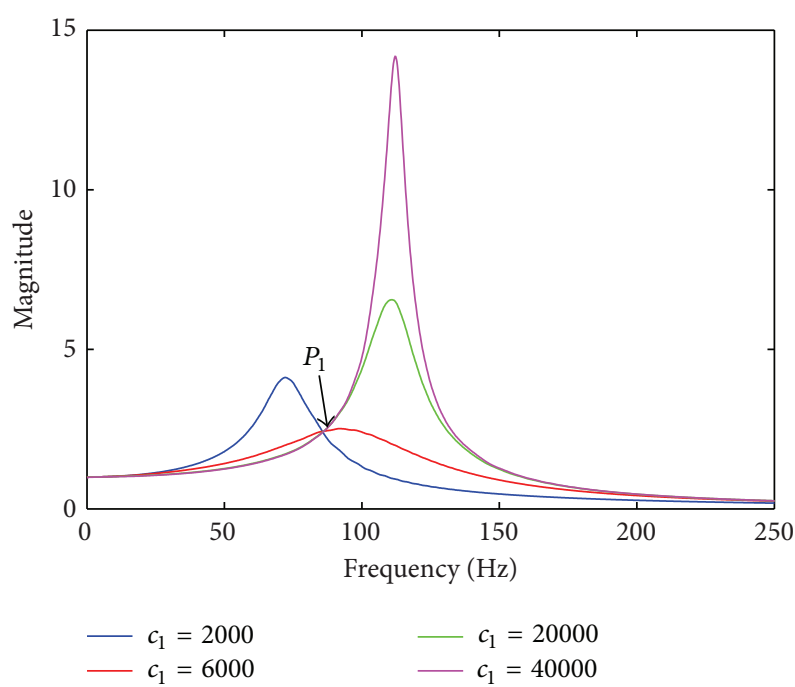

(c)

FIGURE 12: Force transmissibility curves of GNM under $50 \mathrm{~N}$ FE cases. (a) $n_{1}=1.5$; (b) $n_{1}=1$ Maxwell model; (c) $n_{1}=0.85$.

$$
\begin{aligned}
z_{4}^{\prime}=\operatorname{sgn}\left(z_{2}\right)\left|\frac{N_{4} A^{n_{k}-1}\left|z_{2}\right|^{n_{k}}}{\varepsilon_{1}}\right|^{1 / n_{1}}, \\
\begin{aligned}
f\left(z_{2}\right)= & \left(N_{2}+N_{3}\right) z_{2}+N_{4} A^{n_{k}-1} \operatorname{sgn}\left(z_{2}\right)\left|z_{2}\right|^{n_{k}} \\
& -\left[N_{2} z_{0}-\left(N_{2}+N_{3}\right) z_{4}\right]=0,
\end{aligned}
\end{aligned}
$$

where $z_{2}$ can be received by solving the nonlinear equation $f\left(z_{2}\right)=0$, and the corresponding numerical algorithm used in this paper is the secant method. If $n_{1}=n_{k}=1$, taking a differentiation with respect to $\tau$ on both sides of the second formula in (18) simultaneously, then the following equation can be obtained:

$$
\Delta_{1}(\tau)^{\prime \prime}+\Delta_{1}(\tau)+N_{2}\left[\Delta_{1}(\tau)-\Delta_{3}(\tau)\right]=\phi \cos (\lambda \tau),
$$

$$
\begin{aligned}
& N_{2}\left[\Delta_{1}(\tau)^{\prime}-\Delta_{3}(\tau)^{\prime}\right]=N_{3} \Delta_{3}(\tau)^{\prime}+N_{4} \Delta_{2}(\tau)^{\prime}, \\
& N_{4} \Delta_{2}(\tau)=\varepsilon_{1}\left[\Delta_{3}(\tau)^{\prime}-\Delta_{2}(\tau)^{\prime}\right] .
\end{aligned}
$$

Similarly, the form of (20) in phase space is given by

$$
\begin{aligned}
& z_{0}^{\prime}=z_{1}, \\
& z_{1}^{\prime}=-\left(1+N_{2}\right) z_{0}+N_{2} z_{3}+\phi \cos (\lambda \tau), \\
& z_{2}^{\prime}=\frac{N_{2} z_{1}-\left(N_{2}+N_{3}\right)\left[\left(N_{4} / \varepsilon_{1}\right) z_{2}\right]}{N_{2}+N_{3}+N_{4}}, \\
& z_{3}^{\prime}=\frac{N_{2} z_{1}+N_{4}\left[\left(N_{4} / \varepsilon_{1}\right) z_{2}\right]}{N_{2}+N_{3}+N_{4}},
\end{aligned}
$$



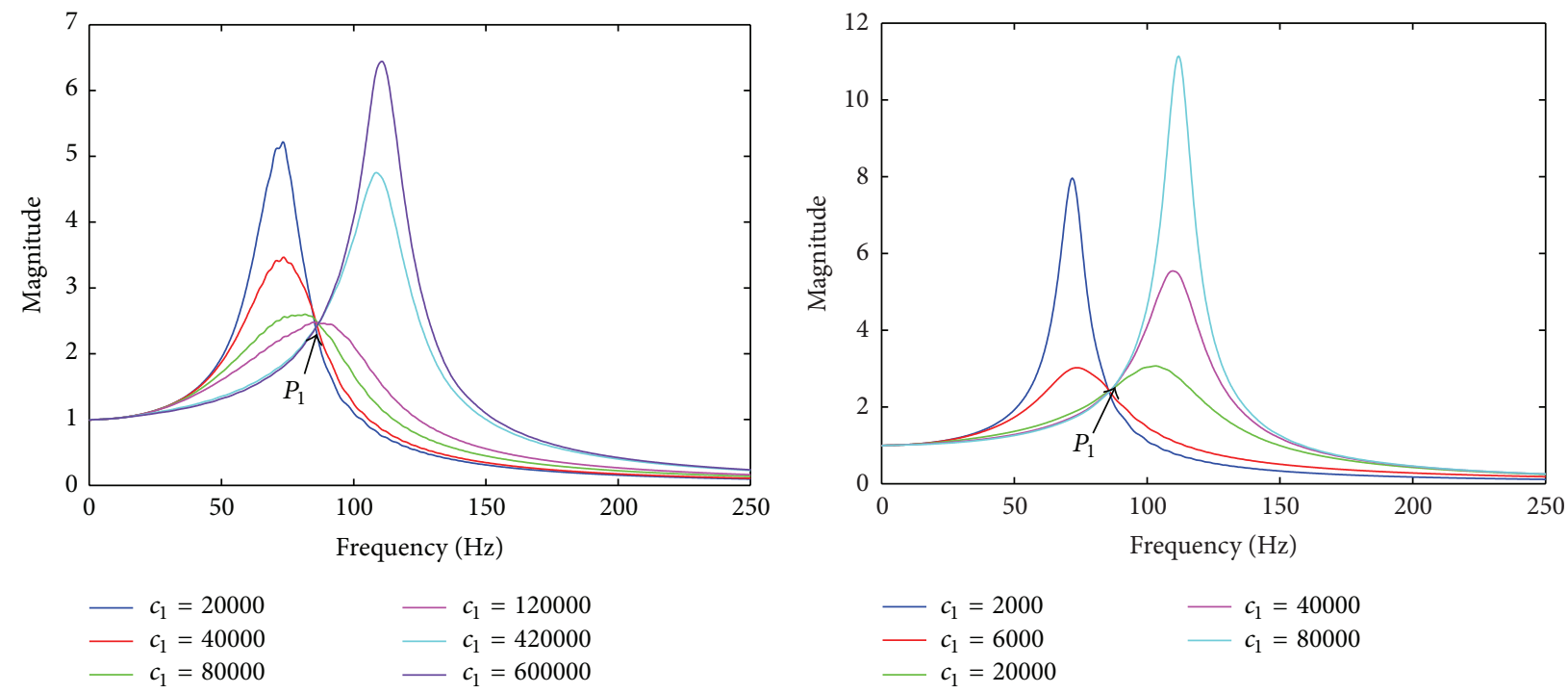

(a)

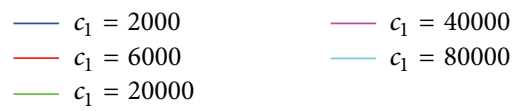

(b)

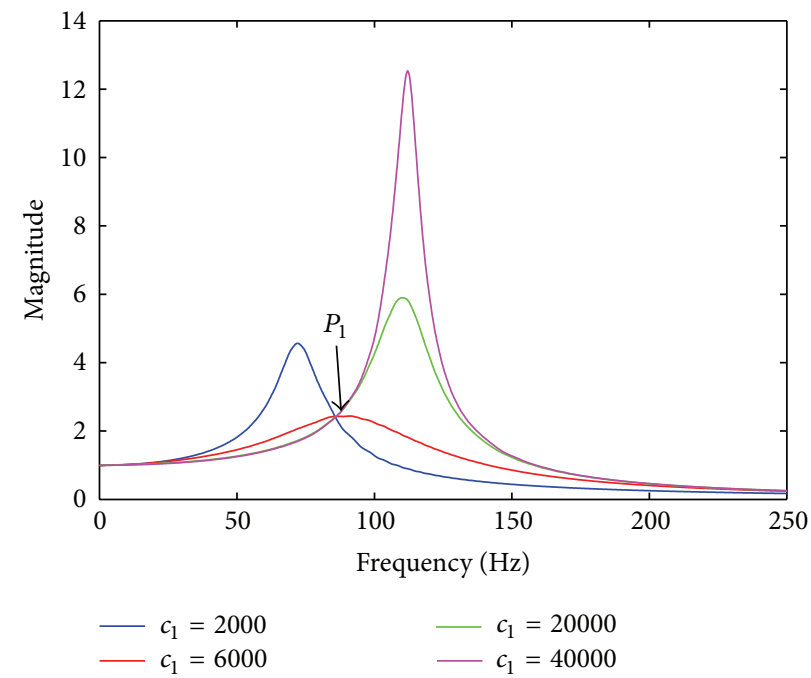

(c)

FIGURE 13: Force transmissibility curves of GNM under $100 \mathrm{~N}$ FE cases. (a) $n_{1}=1.5$; (b) $n_{1}=1$ Maxwell model; (c) $n_{1}=0.85$.

where $\varepsilon_{1}=c_{1} / \sqrt{k_{1} M}$. In terms of the following state vectors and system matrices:

$$
\mathbf{Z}_{2}(\tau)=\left(\begin{array}{l}
z_{0} \\
z_{1} \\
z_{2} \\
z_{3}
\end{array}\right)
$$

$$
\mathbf{T}_{2}(\tau)=\left(\begin{array}{c}
0 \\
\phi \cos (\lambda \tau) \\
0 \\
0
\end{array}\right)
$$

(21) can be written as $\mathbf{Z}_{2}^{\prime}=\mathbf{P}_{2} \mathbf{Z}_{2}+\mathbf{T}_{2}$, whose solution is

$\mathbf{P}_{2}$

$$
=\left(\begin{array}{cccc}
0 & 1 & 0 & 0 \\
-\left(1+N_{2}\right) & 0 & 0 & N_{2} \\
0 & \frac{N_{2}}{N_{2}+N_{3}+N_{4}} & \frac{-\left(N_{2}+N_{3}\right) N_{4}}{\varepsilon_{1}\left(N_{2}+N_{3}+N_{4}\right)} & 0 \\
\frac{N_{2}}{\varepsilon_{2}} & \frac{N_{2}}{N_{2}+N_{3}+N_{4}} & \frac{N_{4}^{2}}{\varepsilon_{1}\left(N_{2}+N_{3}+N_{4}\right)} & 0
\end{array}\right),
$$

$$
\mathbf{Z}_{2}(\tau)=e^{\tau \mathbf{P}_{2}} \mathbf{Z}_{2}(0)+e^{\tau \mathbf{P}_{2}} \int_{0}^{\tau} e^{-u \mathbf{P}_{2}} \mathbf{T}_{2}(u) d u
$$

2.3. Complicated Model. If $c_{1}, c_{2}$, and $k_{4}$ are not equal to zero, (13) can be expressed as

$$
\begin{aligned}
& z_{0}^{\prime}=z_{1}, \\
& z_{1}^{\prime}=-\left(1+N_{2}\right) z_{0}+N_{2} z_{3}+\phi \cos (\lambda \tau),
\end{aligned}
$$



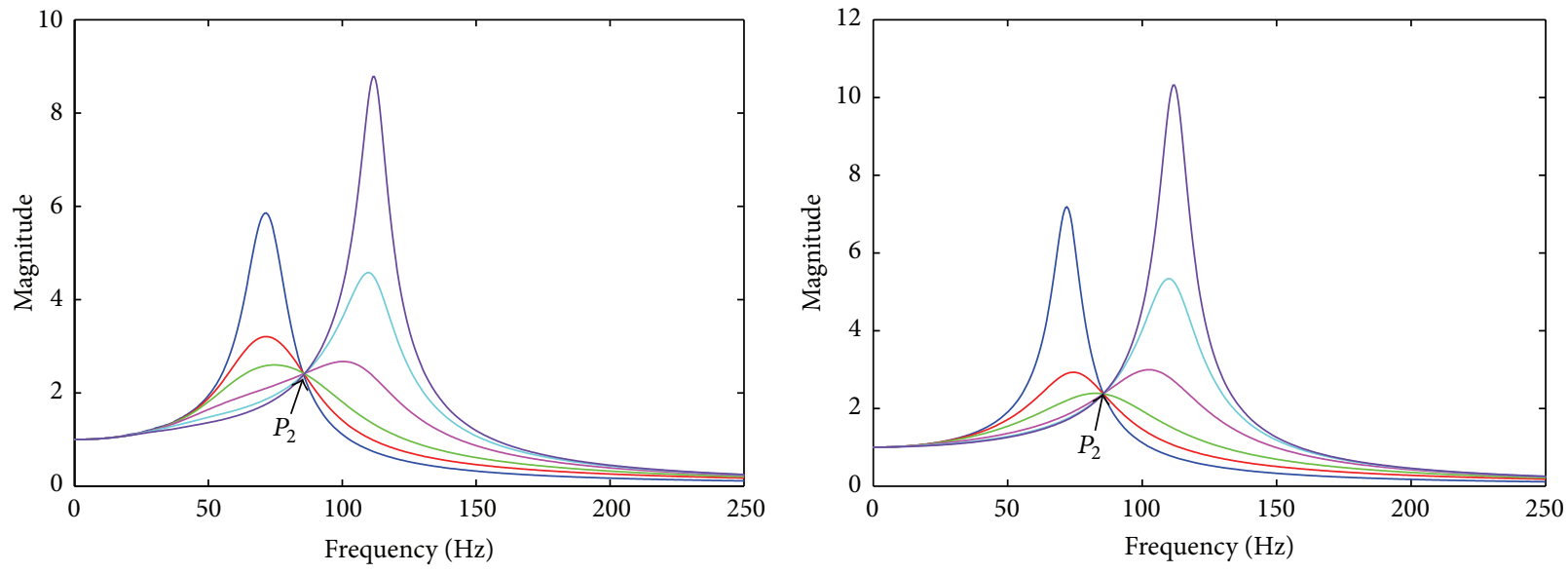

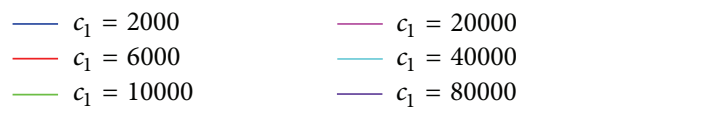

(a)

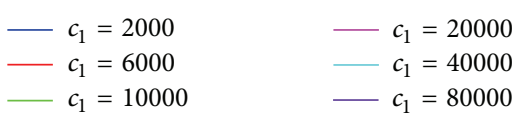

(b)
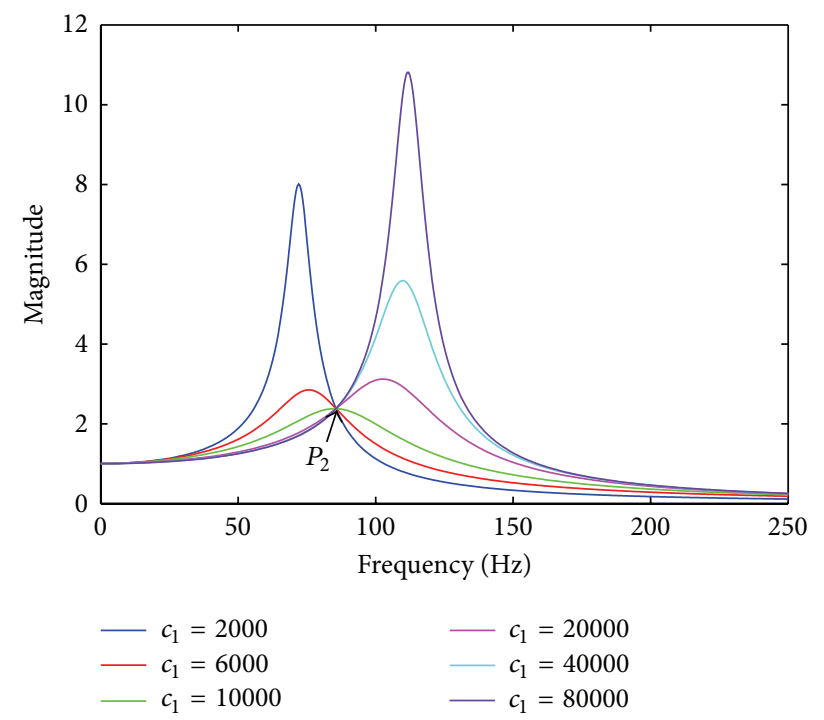

(c)

FIGURE 14: Absolute displacement transmissibility curves of GNM under $0.76 \mathrm{~mm}$ FDE cases. (a) $n_{1}=1.5$; (b) $n_{1}=1$ Maxwell model; (c) $n_{1}=0.85$.

$$
\begin{aligned}
z_{2}^{\prime}= & \operatorname{sgn}\left(D_{0}\right) \times\left|D_{0}\right|^{1 / n_{2}} \\
& \quad-\operatorname{sgn}\left(z_{2}\right)\left|\frac{N_{4} A^{n_{k}-1}\left|z_{2}\right|^{n_{k}}}{\varepsilon_{1}}\right|^{1 / n_{1}}, \\
z_{3}^{\prime}= & \operatorname{sgn}\left(D_{0}\right) \times\left|D_{0}\right|^{1 / n_{2}}
\end{aligned}
$$

$$
\begin{gathered}
z_{2}^{\prime}=\frac{1}{\varepsilon_{1} \varepsilon_{2}}\left[\varepsilon_{1} N_{2} z_{0}-\left(\varepsilon_{1} N_{4}+\varepsilon_{2} N_{4}\right) z_{2}\right. \\
\left.-\left(\varepsilon_{1} N_{2}+\varepsilon_{1} N_{3}\right) z_{3}\right], \\
z_{3}^{\prime}=\frac{1}{\varepsilon_{2}}\left[N_{2} z_{0}-N_{4} z_{2}-\left(N_{2}+N_{3}\right) z_{3}\right],
\end{gathered}
$$

where $D_{0}=\left(N_{2} z_{0}-N_{4} A^{n_{k}-1} \operatorname{sgn}\left(z_{2}\right)\left|z_{2}\right|^{n_{k}}-\left(N_{2}+N_{3}\right) z_{3}\right) / \varepsilon_{2}$. If $n_{1}=n_{2}=n_{k}=1$, the system becomes a linear one and (24) can be simplified as

$$
\begin{aligned}
& z_{0}^{\prime}=z_{1}, \\
& z_{1}^{\prime}=-\left(1+N_{2}\right) z_{0}+N_{2} z_{3}+\phi \cos (\lambda \tau),
\end{aligned}
$$

where $\varepsilon_{1}=c_{1} / \sqrt{k_{1} M}, \varepsilon_{2}=c_{2} / \sqrt{k_{1} M}$. By setting

$$
\mathbf{Z}_{3}(\tau)=\left(\begin{array}{l}
z_{0} \\
z_{1} \\
z_{2} \\
z_{3}
\end{array}\right)
$$



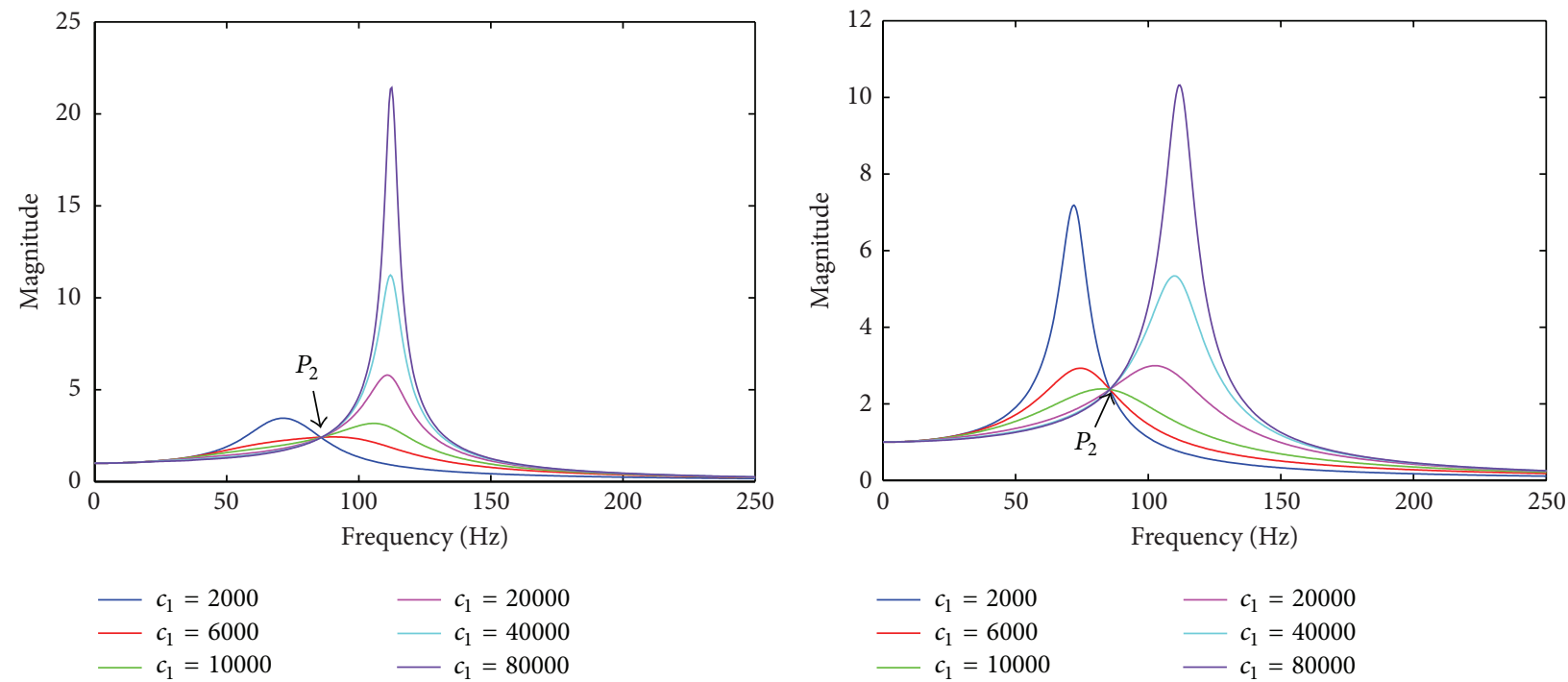

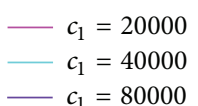

(a)

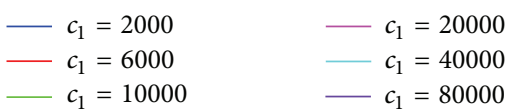

(b)
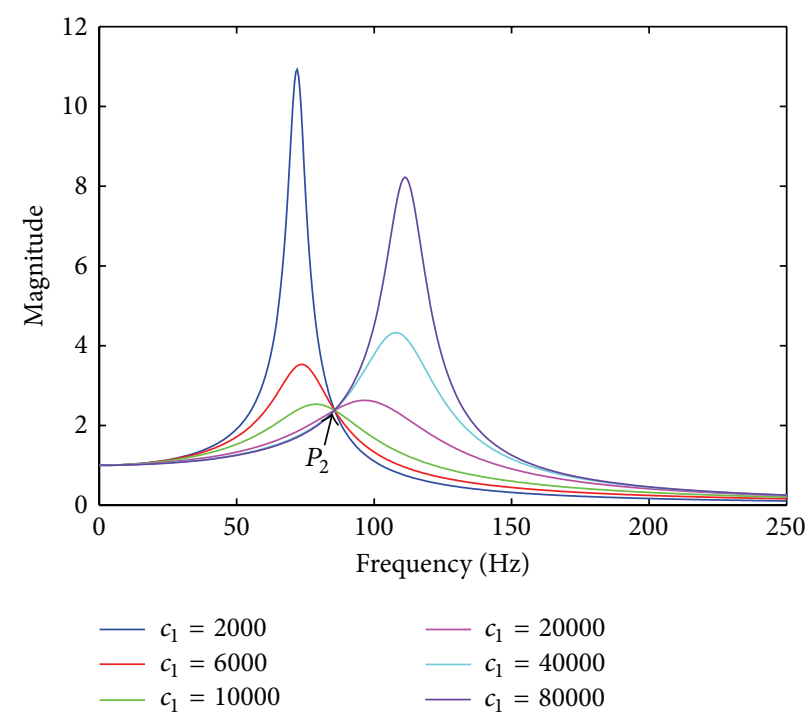

(c)

Figure 15: Absolute displacement transmissibility curves of GNM under $5.07 \mathrm{~mm}$ FDE cases. (a) $n_{1}=1.5$; (b) $n_{1}=1$ Maxwell model; (c) $n_{1}=0.85$.

$\mathbf{P}_{3}$

$$
\begin{gathered}
=\left(\begin{array}{cccc}
0 & 1 & 0 & 0 \\
-\left(1+N_{2}\right) & 0 & 0 & N_{2} \\
\frac{N_{2}}{\varepsilon_{2}} & 0 & -\frac{\varepsilon_{1} N_{4}+\varepsilon_{2} N_{4}}{\varepsilon_{1} \varepsilon_{2}} & -\frac{\varepsilon_{1} N_{2}+\varepsilon_{1} N_{3}}{\varepsilon_{1} \varepsilon_{2}} \\
\frac{N_{2}}{\varepsilon_{2}} & 0 & -\frac{N_{4}}{\varepsilon_{2}} & -\frac{N_{2}+N_{3}}{\varepsilon_{2}}
\end{array}\right), \\
\mathbf{T}_{3}(\tau)=\left(\begin{array}{c}
0 \\
\phi \cos (\lambda \tau) \\
0 \\
0
\end{array}\right),
\end{gathered}
$$

(25) reduces to $\mathbf{Z}_{3}^{\prime}=\mathbf{P}_{3} \mathbf{Z}_{3}+\mathbf{T}_{3}$, and the corresponding solution is

$$
\mathbf{Z}_{3}(\tau)=e^{\tau \mathbf{P}_{3}} \mathbf{Z}_{3}(0)+e^{\tau \mathbf{P}_{3}} \int_{0}^{\tau} e^{-u \mathbf{P}_{3}} \mathbf{T}_{3}(u) d u
$$

2.4. Solving Method and System Output. In the cases of FE, $\phi=1$, the mainly considered index is the force $F_{T}$ which is transmitted into the foundation and it can be written as

$$
F_{T}=F(t)-M \ddot{x}_{p}=k_{1} x_{p}+k_{2}\left(x_{p}-x\right) .
$$




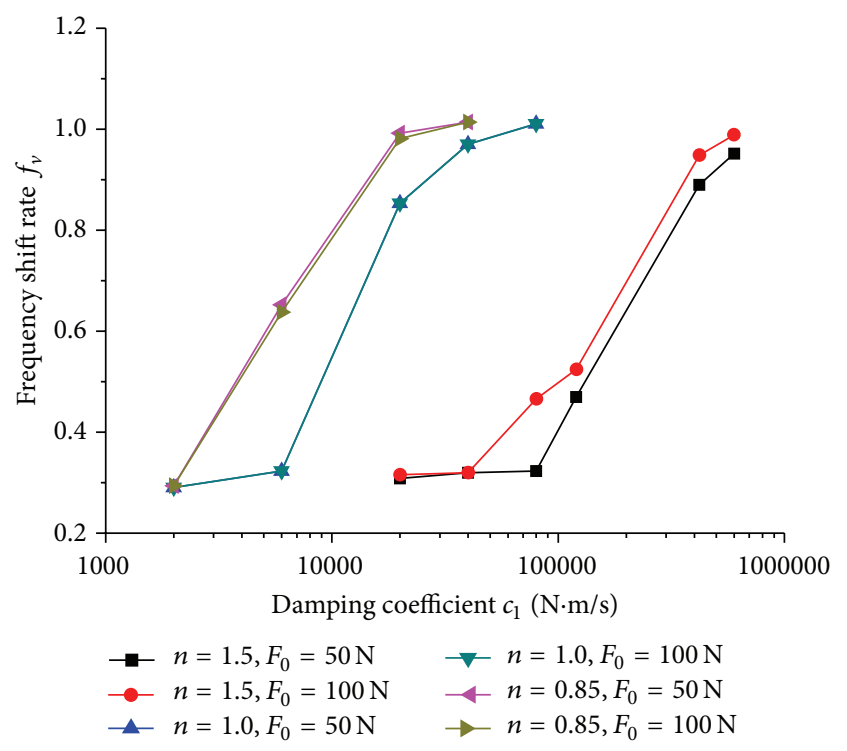

(a)

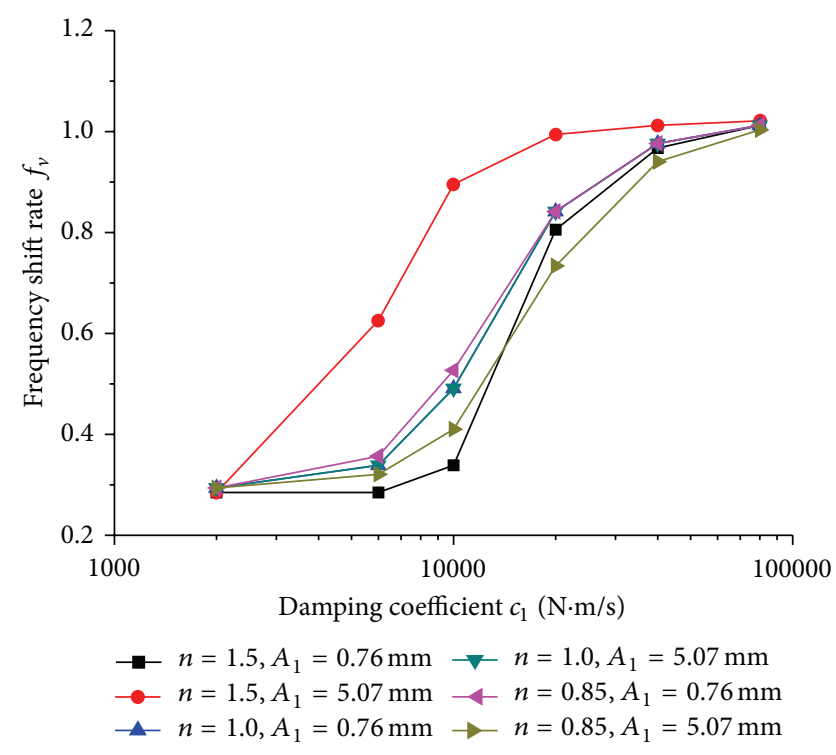

(b)

FIGURE 16: Frequency shift rates of GNM. (a) FE cases; (b) FDE cases.

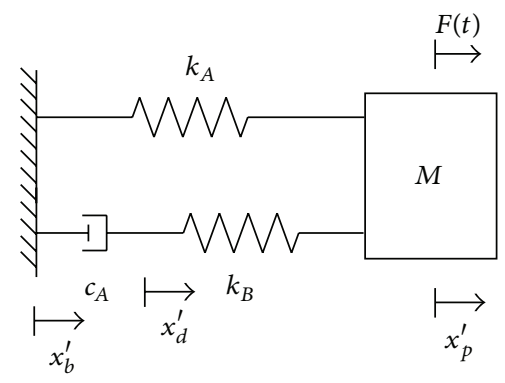

FIGURE 17: Three-parameter model.

The nondimensional form of this index is given by

$$
\begin{aligned}
\Gamma(\tau) & =\phi \cos (\lambda \tau)-\Delta_{1}(\tau)^{\prime \prime} \\
& =\Delta_{1}(\tau)+N_{2}\left[\Delta_{1}(\tau)-\Delta_{3}(\tau)\right] \\
& =z_{0}+N_{2}\left(z_{0}-z_{3}\right) .
\end{aligned}
$$

However, in the cases of FDE, $\phi=\lambda^{2}$, the mainly considered indices are the relative displacement $R=x_{p}-x_{b}$ and absolute displacement $x_{p}$; the corresponding nondimensional forms are given by

$$
\begin{gathered}
\Delta_{1}(\tau)=z_{0}, \\
\Lambda=\Delta_{1}(\tau)+\cos (\lambda \tau) \\
=z_{0}+\cos (\lambda \tau) .
\end{gathered}
$$

Moreover, the fourth-order Runge-Kutta method is applied to obtain the numerical solutions of (15), (19), and (24), and, with the use of secant method, the variable $z_{2}$ in (19) can be obtained through the way to solve the nonlinear equation $f\left(z_{2}\right)=0$ in each iterative step.

\section{Test and Simulation}

3.1. Test Setup of Hysteretic Loops. As illustrated in Figure 5, the test setup of hysteretic loops is mainly composed of load bearing fixture, force transducer, laser displacement transducer, shaker, data acquisition and analysis system, control system, power amplifier, and so forth. Firstly, the sinusoidal signal, which is produced by the control system and has a prescribed frequency, is amplified through the power amplifier and then transmitted to the shaker; thus the whole system is excited. The right end of the isolator is connected to the shaker, and the displacement of this end is measured by the laser displacement transducer (Germany Polytec laser displacement measurement system, whose type is psv-200 and its displacement accuracy is $0.1 \mu \mathrm{m})$. Besides, the other end of the isolator is connected to the load bearing fixture through a force transducer. The force and displacement signals are sampled by the data acquisition and analysis system, and then the hysteretic loops between them at this frequency can be obtained. After changing the excitation frequency and repeating the previous steps again, the hysteretic loops at every frequency can be easily received. 


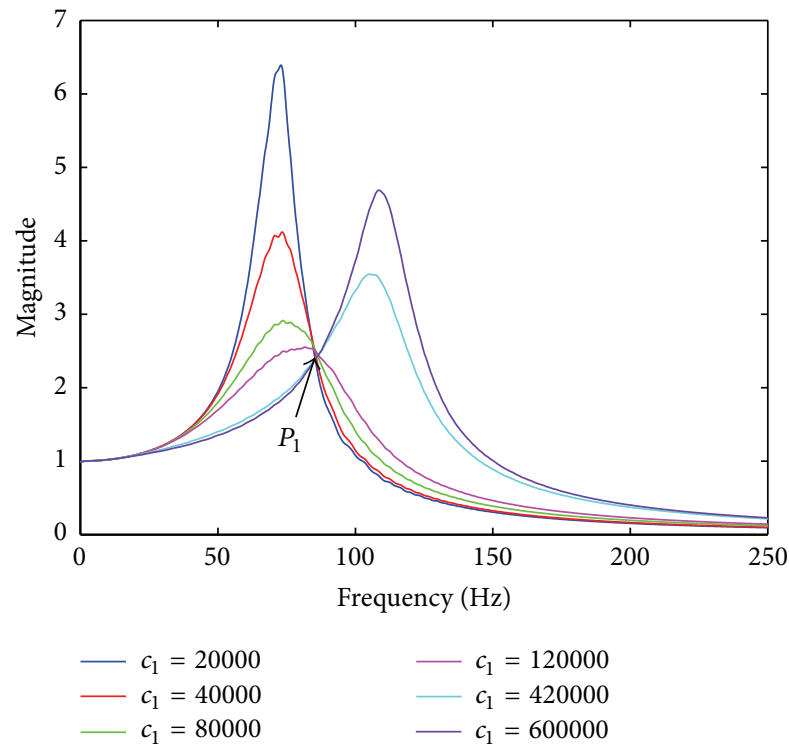

(a)

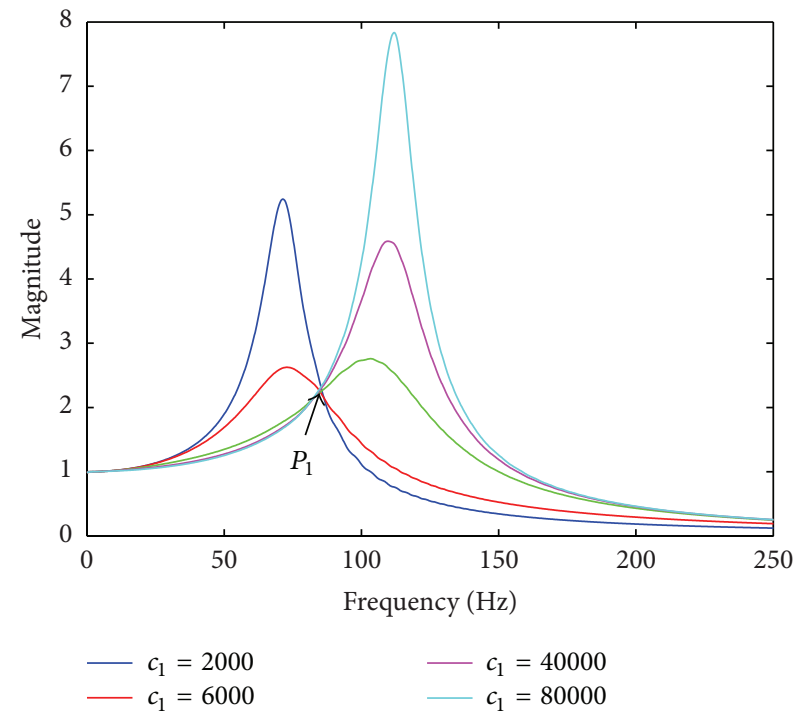

(b)

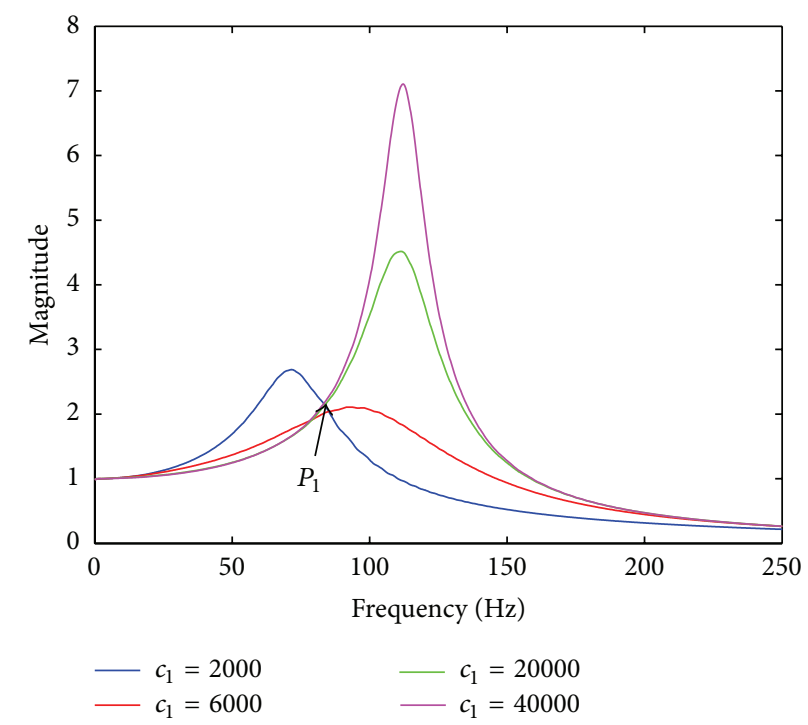

(c)

FIGURE 18: Force transmissibility curves of CM under $50 \mathrm{~N}$ FE cases. (a) $n_{1}=1.5$; (b) $n_{1}=1$; (c) $n_{1}=0.85$.

During the test, the whole system is connected to ground to eliminate the effects of electric noise, and a band-pass filter is used to remove the DC component and other interfering frequencies. Figure 6 is a picture of the test setup.

3.2. Test Setup of Force Transmissibility. The test setup of force transmissibility is shown in Figure 7; it mainly contains control system, power amplifier, shaker, large mass, load bearing fixture, isolator, force transducer, data acquisition and analysis system, and so forth. The large mass and the shaker are suspended from the ceiling. First of all, a sine sweep signal produced by the control system is amplified by the power amplifier and then sent to the shaker; thus the whole system is excited. The large mass is connected to the shaker through a force transducer B, and its left end is next to the isolator. Moreover, the left end of the isolator is connected to the load bearing fixture through a force transducer $\mathrm{A}$; thus the displacement at this end is constrained. The two force signals are sampled by the data acquisition and analysis system, and the corresponding transmissibility curves can be obtained after data processing.

3.3. Identification of Nonlinear Model Parameters. For the isolator studied in this paper, $n_{1}=n_{2}=0.85$, the parameters of vibration models of Section 2 , that is, $c_{1}, c_{2}, k_{4}$, and $n_{k}$, should be identified through the hysteretic loops of test at 


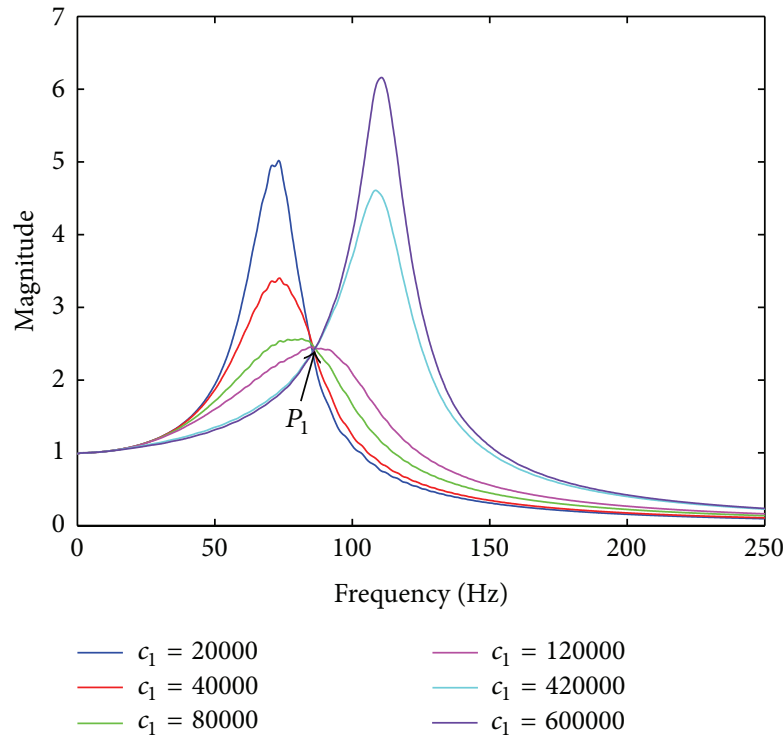

(a)

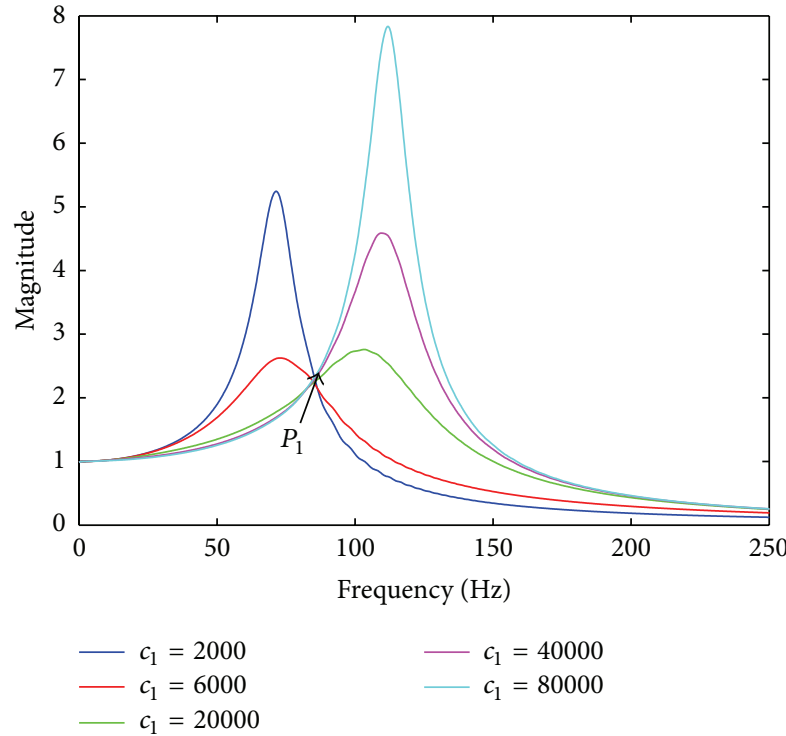

(b)

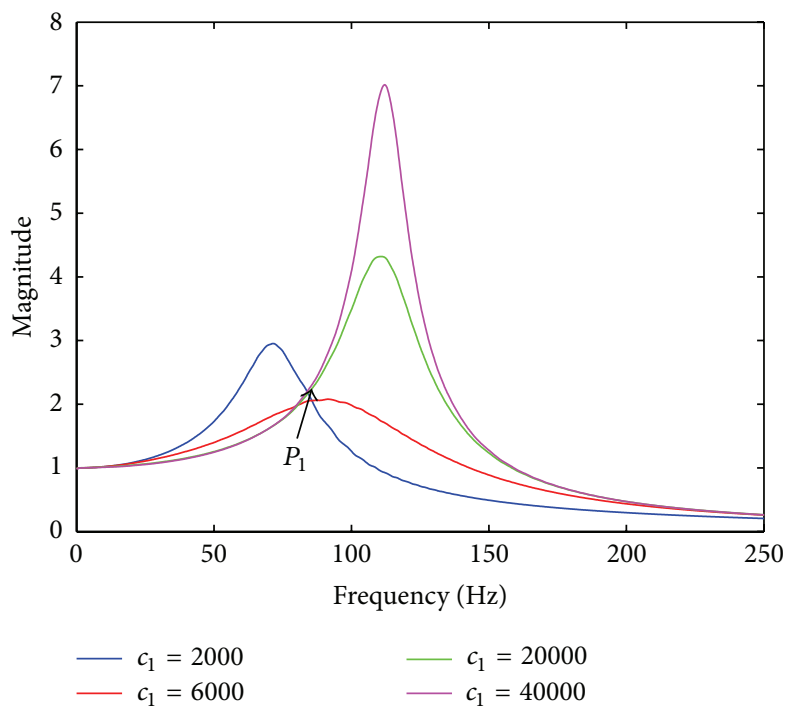

(c)

FIGURE 19: Force transmissibility curves of CM under $100 \mathrm{~N}$ FE cases. (a) $n_{1}=1.5$; (b) $n_{1}=1$; (c) $n_{1}=0.85$.

every frequency. The objective function in the FE cases is defined as

$$
\text { Minimize } J=\sqrt{\frac{1}{N} \sum_{i=1}^{N}\left(x_{p, \text { the }}[i]-x_{p \text {,exp }}[i]\right)^{2}}
$$

where $N$ is number of comparative points, $x_{p \text {,the }}$ and $x_{p \text {,exp }}$ are the absolute displacements of simulation and test, respectively. Moreover, the GPS algorithm of MATLAB optimization toolbox is adopted in this paper. As there is no need of the gradient and derivative of objective function, hence, it is suitable for the complex optimization problems, which have nondifferentiable or even noncontinuous objective functions. During each iterative step, the objective function values of current point and its surrounding mesh points are calculated; if one point has the minimum value, then this point will become the current point in next iterative step. Besides, the size of mesh is also changeable; if the current point has the lowest value, then the size of mesh will decrease in the next iterative step; contrarily, the size of mesh will increase if the lowest value is at other mesh points. Since the mass of isolator and the corresponding inertial force can be ignored, the amplitude of excitation force of simulation is set equal to that of force transducer of test. Further, the performance of isolator changes with excitation frequency significantly; thus the identified procedure is executed at every frequency point. Figure 8 shows the variations of each parameter with excitation frequency.

Since viscous fluid is included in the isolator, thus different flow states arise at different frequencies. As illustrated 


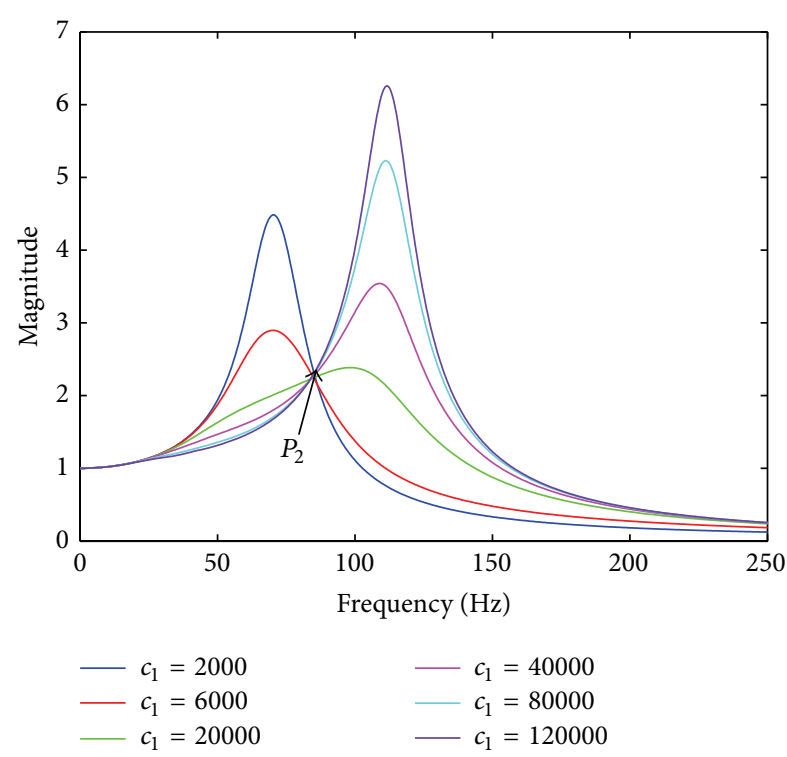

(a)

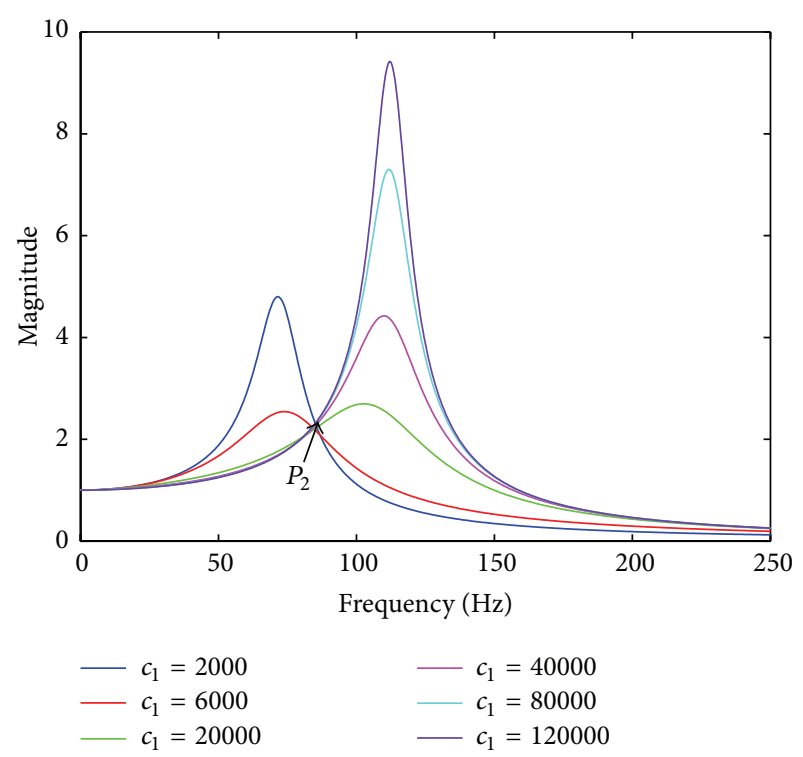

(b)

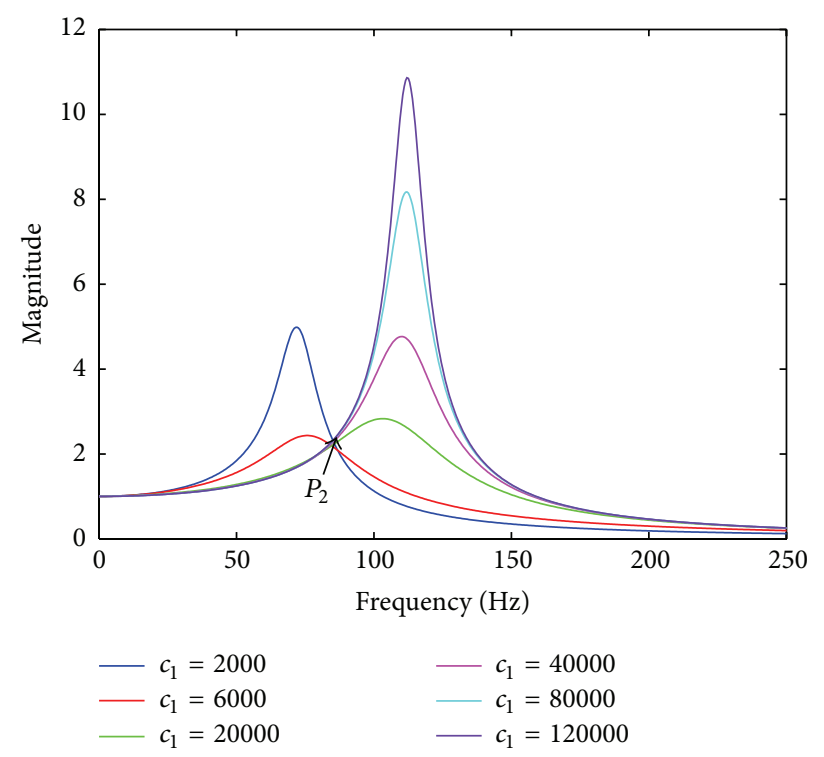

(c)

Figure 20: Absolute displacement transmissibility curves of CM under $0.76 \mathrm{~mm}$ FDE cases. (a) $n_{1}=1.5$; (b) $n_{1}=1$; (c) $n_{1}=0.85$.

in Figure 8, the stiffness exponent $n_{k}$ is always equal to one except some specific frequency points; thus the effect of nonlinear stiffness is not obvious. On the contrary, the variation ranges of other parameters are very wide, so the performance of isolator is directly related to excitation frequency.

3.4. Validation of Models. If $n_{1}=n_{k}=1$, a linear Maxwell model can be simplified from the GNM, and the corresponding theoretical parameter values are given by [24]. Each identified parameter is reentered into the simulation model, and then the hysteretic loops of simulation are compared with those of test, as shown in Figure 9.
As shown in Figure 9, the hysteretic loops are numerically integrated with the use of trapezoidal formula, and the relative area ratio $\eta$ between simulation and test can be expressed as

$$
\eta=\left|\frac{W_{\text {the }}-W_{\exp }}{W_{\exp }}\right| \times 100 \%,
$$

where $W_{\text {the }}$ and $W_{\text {exp }}$ are the average areas of hysteretic loops of simulation and test, respectively. Figure 10 shows the variations of $\eta$ with different frequencies.

It can be seen that the simple model cannot accurately characterize the practical vibration of isolator because of its large value of $\eta$. The GNM and CM have a smaller $\eta$ and 


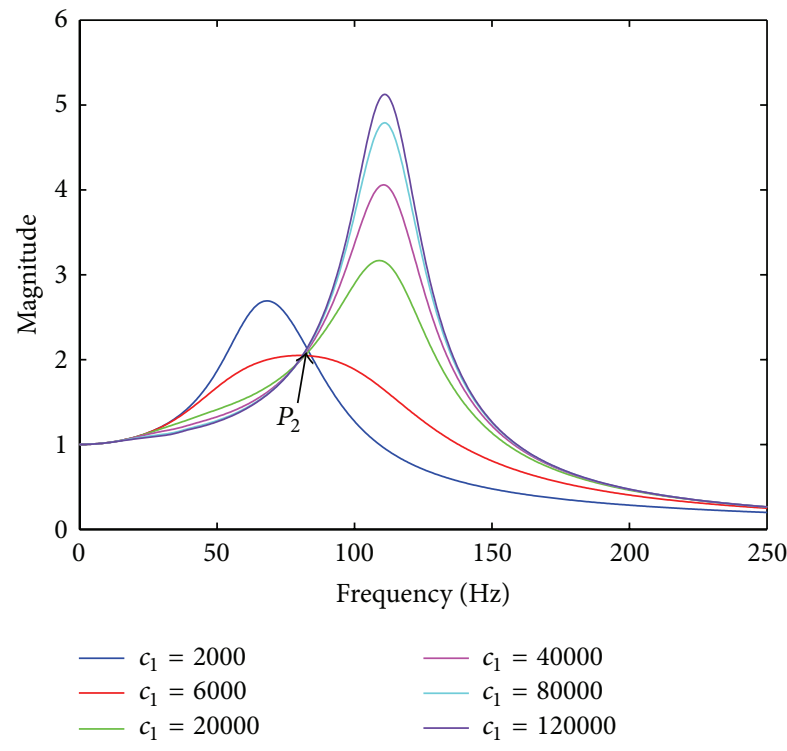

(a)

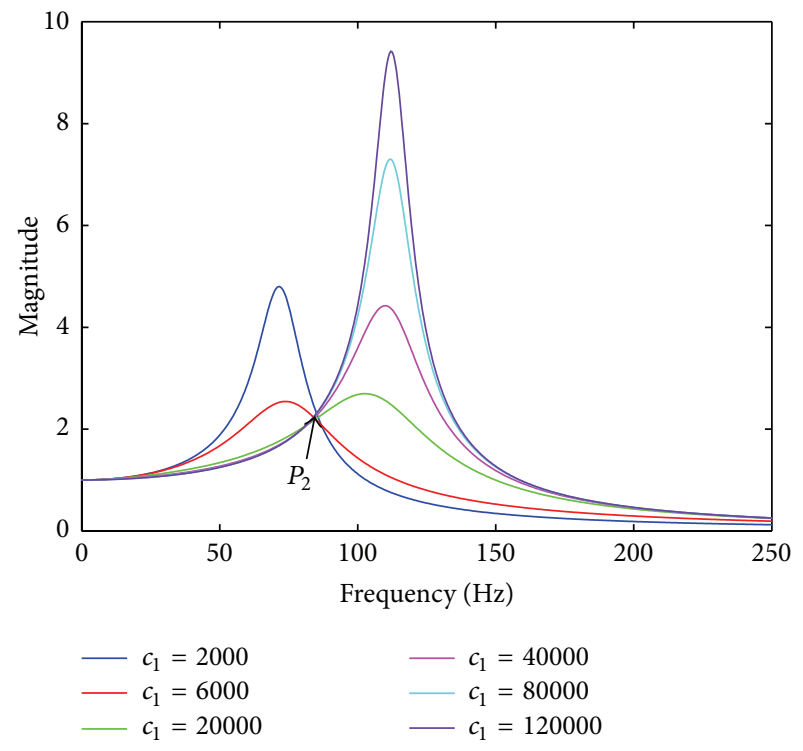

(b)

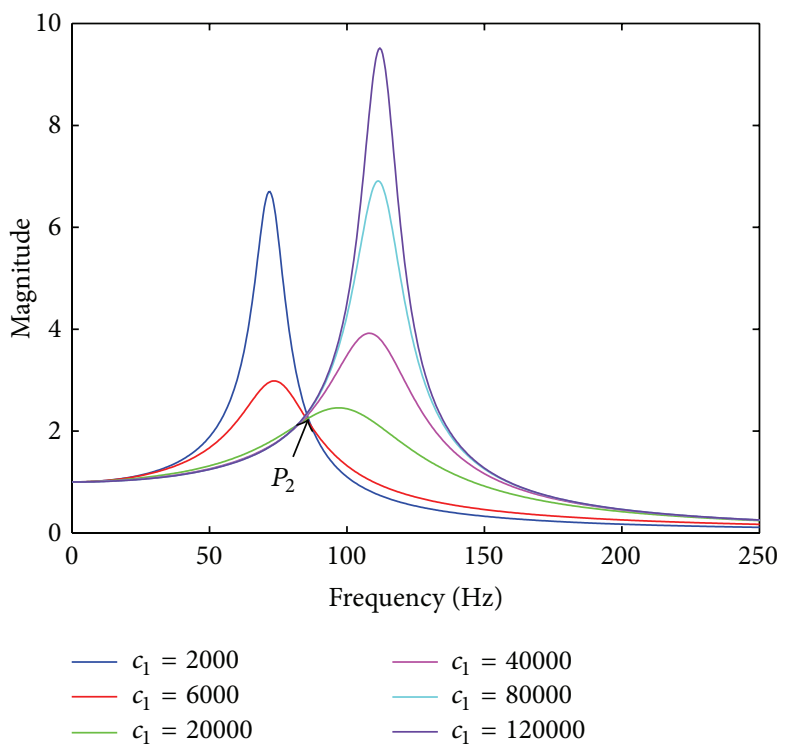

(c)

FIgURE 21: Absolute displacement transmissibility curves of CM under $5.07 \mathrm{~mm}$ FDE cases. (a) $n_{1}=1.5 ;$ (b) $n_{1}=1 ;$ (c) $n_{1}=0.85$.

are superior to the Maxwell model in the frequency range from $5 \mathrm{~Hz}$ to $20 \mathrm{~Hz}$. However, the Maxwell model has more advantages at high frequencies. Moreover, the differences of the value of $\eta$ between GNM and CM are little, while the GNM is simpler than the other one. Since the equality of amplitude of excitation force between simulation and test has been achieved, large elastic deformation of fluid reservoir occurs in the low frequency band; thus the nonlinear effect of fluid becomes strong, and the GNM and CM can properly represent the physical vibration of isolator. However, in the high frequency band, the Maxwell model has more excellent performance because of the small elastic deformation of fluid reservoir. Further, the curves of Figure 8 are entered into the simulation model, and the corresponding force transmissibility curves are shown in Figure 11.
As illustrated in Figure 11, the fundamental frequency of the transmissibility curves of simulation is $2.41 \%$ larger than that of experiment, and the relative magnification factors of the Maxwell model, GNM, and CM are $+2.00 \%,-12.34 \%$, and $-22.89 \%$, respectively. Thus the performance of Maxwell model is more outstanding; the reason why this happens may be that the GNM and CM are only effective in the range of 5$20 \mathrm{~Hz}$, while the Maxwell model is effective in a more broad range of $30-200 \mathrm{~Hz}$. The second peak at $157 \mathrm{~Hz}$ may be caused by the error of curve fitting.

\section{Parametric Analysis of Nonlinear Models}

First, we assume that each model parameter is mutually independent, and the interactive effect of these parameters 


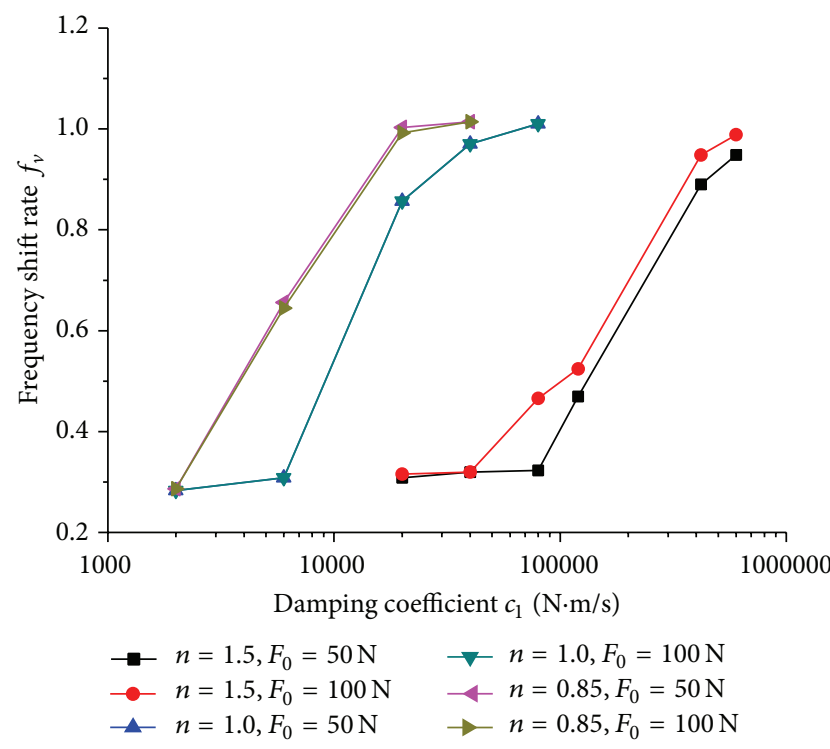

(a)

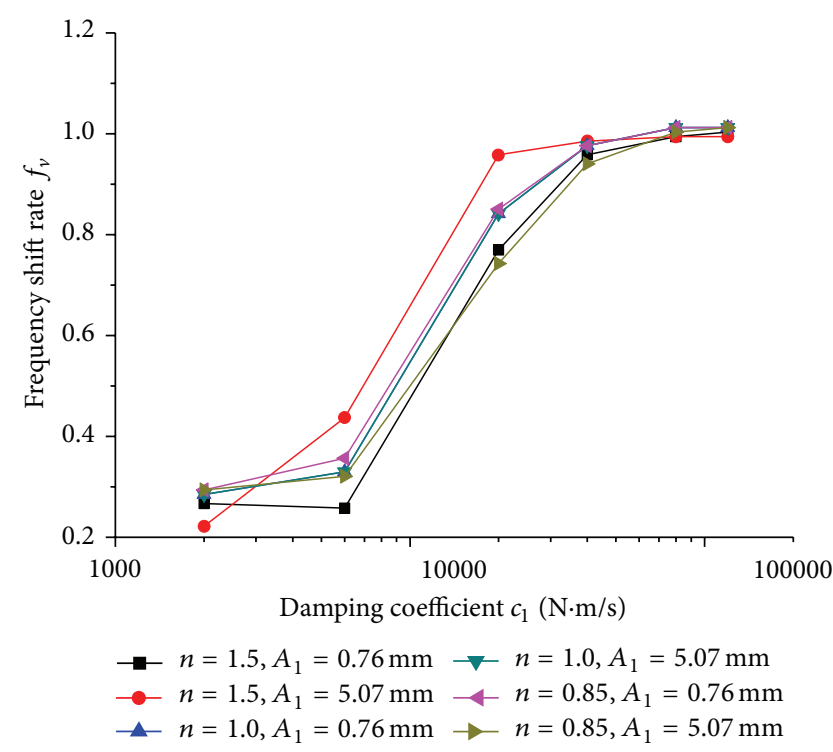

(b)

FIGURE 22: Frequency shift rates of CM. (a) FE cases; (b) FDE cases.

TABLE 1: Table head of $\mathrm{L}_{25}(6)$.

\begin{tabular}{|c|c|c|c|c|c|c|c|c|}
\hline Factors & $c_{1}$ & $n_{1}$ & $c_{2}$ & $n_{2}$ & $k_{4}$ & $n_{k}$ & Fundamental frequency & Magnification factor \\
\hline Number & 1 & 2 & 3 & 4 & 5 & 6 & & \\
\hline \multicolumn{9}{|l|}{ Test 1} \\
\hline \multicolumn{9}{|l|}{ Test 2} \\
\hline Test... & & & & & & & & \\
\hline
\end{tabular}

TABLE 2: Levels of each nonlinear parameter.

\begin{tabular}{lccccc}
\hline & $c_{1} /(\mathrm{N} \cdot \mathrm{s} / \mathrm{m})$ & $n_{1}$ & $c_{2} /(\mathrm{N} \cdot \mathrm{s} / \mathrm{m})$ & $n_{2}$ & $k_{4} /(\mathrm{N} / \mathrm{m})$ \\
\hline Level 1 & 2000 & 0.30 & 2000 & 0.30 & $3.824 \times 10^{6}$ \\
Level 2 & 20000 & 0.50 & 20000 & 0.50 & $8.928 \times 10^{6}$ \\
Level 3 & 40000 & 0.85 & 40000 & 0.85 & $1.000 \times 10^{7}$ \\
Level 4 & 80000 & 1.00 & 80000 & 1.00 & $2.000 \times 10^{7}$ \\
Level 5 & 120000 & 1.50 & 120000 & 1.50 & $2.950 \times 10^{7}$ \\
\hline
\end{tabular}

is neglected; then the orthogonal test design method is adopted. Table 1 shows the design of table head of $\mathrm{L}_{25}(6)$, which is applied to investigate the effects of nonlinear model parameters on the transmissibility.

Table 2 shows the levels of each nonlinear parameter.

After the simulation of each test in orthogonal Table 1 and the analysis of range, it can be known that the flow index and damping coefficient are two important factors of microvibration isolation. Then the following parameter values, that is, $n_{k}=1$ and $k_{4}=1.000 \times 10^{7} \mathrm{~N} / \mathrm{m}$, are adopted under a comprehensive consideration of fundamental frequency, magnification factor, and roll-off performance in high frequency band. Other standard values used in this parametric analysis are $k_{1}=3.669 \times 10^{6} \mathrm{~N} / \mathrm{m}, k_{2}=1.191 \times$ $10^{8} \mathrm{~N} / \mathrm{m}, k_{3}=2.482 \times 10^{6} \mathrm{~N} / \mathrm{m}, c_{1}=20000 \mathrm{~N} \cdot \mathrm{s} / \mathrm{m}$, and $c_{2}=$ $1000 \mathrm{~N} \cdot \mathrm{s} / \mathrm{m}$. Moreover, in order to evaluate the performance of vibration isolation, an index called frequency shift rate $f_{v}$ is also defined as follows:

$$
\begin{gathered}
f_{v}=\frac{f-f_{0}}{f_{0}}, \\
f_{0}=\frac{1}{2 \pi} \sqrt{\frac{k_{1}}{M}},
\end{gathered}
$$

where $f$ is the frequency at which the resonant peak of transmissibility occurs.

4.1. Generalized Nonlinear Model. Figures 12, 13, 14, and 15 show the effects of flow index $n_{1}$ and damping coefficient $c_{1}$ on the performance of GNM in different excitation cases.

As shown in Figures 12, 13, 14, and 15, the system contains two resonant regions, and all of the transmissibility curves 


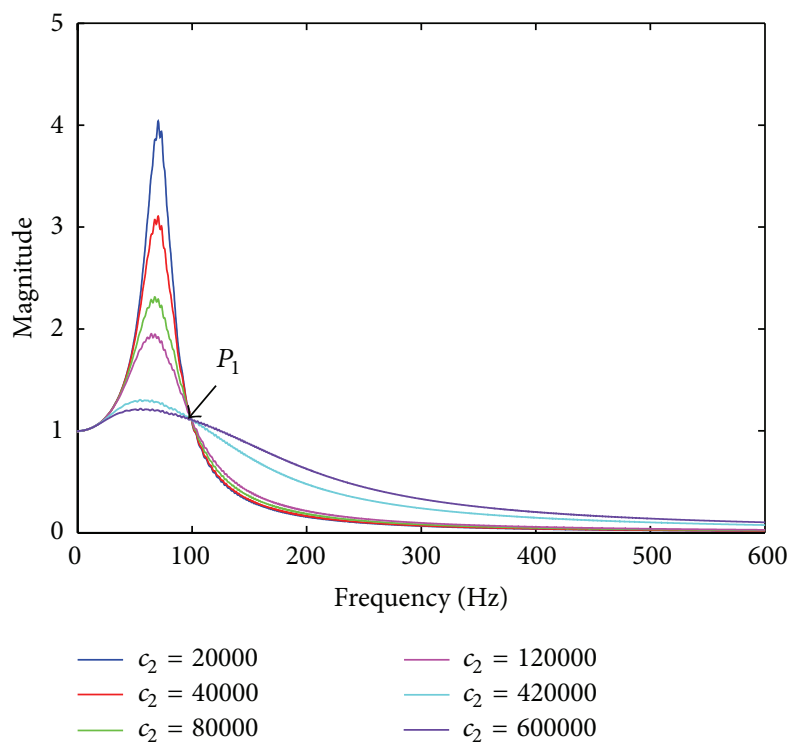

(a)

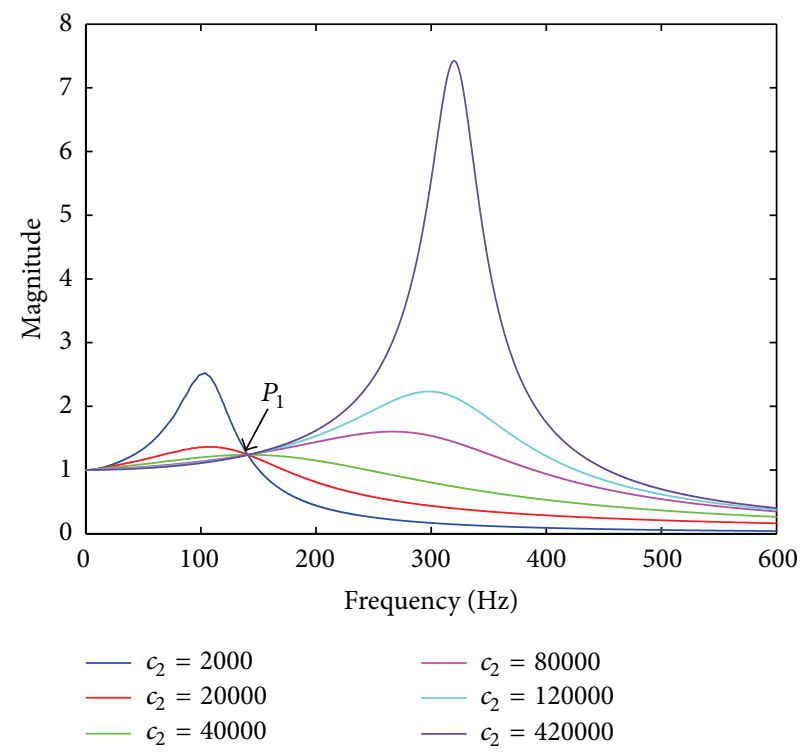

(b)
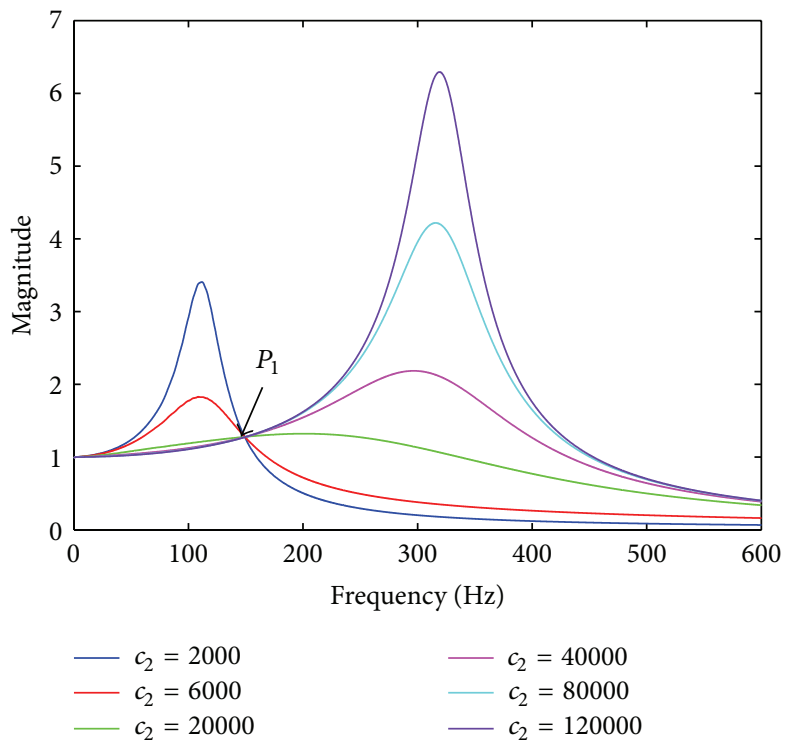

(c)

FIGURE 23: Force transmissibility curves of CM under $50 \mathrm{~N} \mathrm{FE} \mathrm{cases.} \mathrm{(a)} n_{1}=1.5$; (b) $n_{1}=1$; (c) $n_{1}=0.85$.

pass through one common point $P_{1}$ or $P_{2}$, at which there is the lowest resonant peak and it can be the critical point between the first resonant region and the second resonant region. With the increase of damping coefficient $c_{1}$, the fundamental frequency of system transfers from the first resonant region to the second resonant region, while the resonant peak firstly reduces to $P_{1}$ or $P_{2}$ point and then increases with the raise of damping coefficient $c_{1}$. Furthermore, in the first resonant region, the smaller the damping coefficient $c_{1}$ is, the larger the high frequency roll-off rate is, but there is an opposite situation in the second resonant region. When the frequency is larger than $150 \mathrm{~Hz}$, the high frequency roll-off rates under different damping coefficients $c_{1}$ become consistent with each other. Figure 16 shows the frequency shift rates of GNM under different excitation cases.
As indicated in Figure 16, in the cases of FE, the $n_{1}=1$ Maxwell model and the $n_{1}=0.85 \mathrm{GNM}$ have high values of $f_{v}$; thus the fundamental frequency is easy to transfer from the first resonant region to the second one. However, the $n_{1}=$ 1.5 GNM keeps a wide range of damping coefficient $c_{1}$ into the first resonant region. In the cases of $0.76 \mathrm{~mm}$ FDE, the values of $f_{v}$ of $n_{1}=1.5 \mathrm{GNM}$ are slightly lower than those of others, and the distinction of these three nonlinear models is very small; thus the performance of isolator is mainly determined by the damping coefficient $c_{1}$. However, an opposite situation occurs in the cases of $5.07 \mathrm{~mm}$ FDE, the values of $f_{v}$ of $n_{1}=1.5 \mathrm{GNM}$ are much higher than those of others, and the damping exponent has significant effects on the performance of vibration isolation. Moreover, if $n_{1}=1$, the linear Maxwell model has the same transmissibility curves even though 


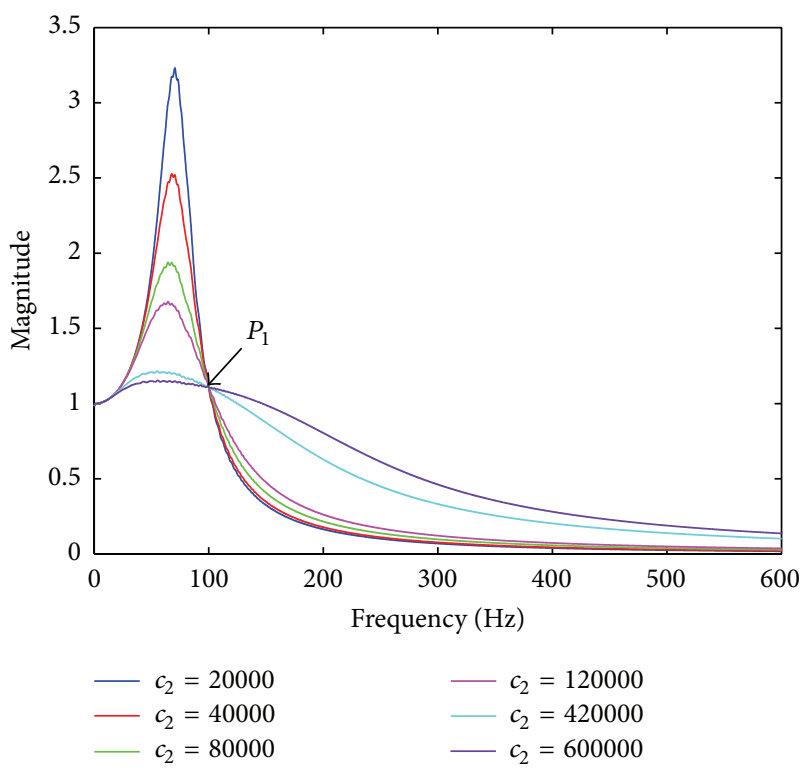

(a)
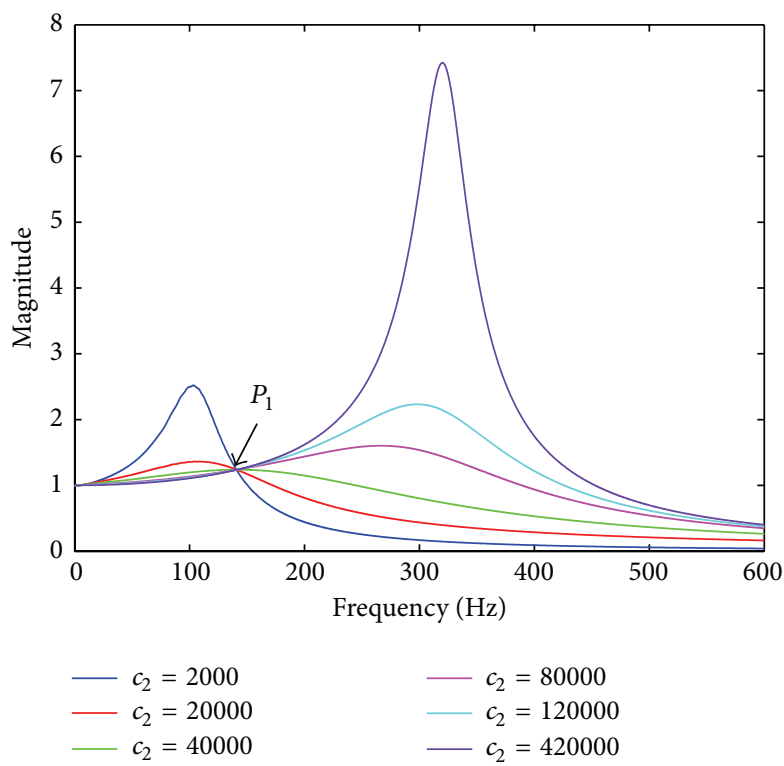

(b)

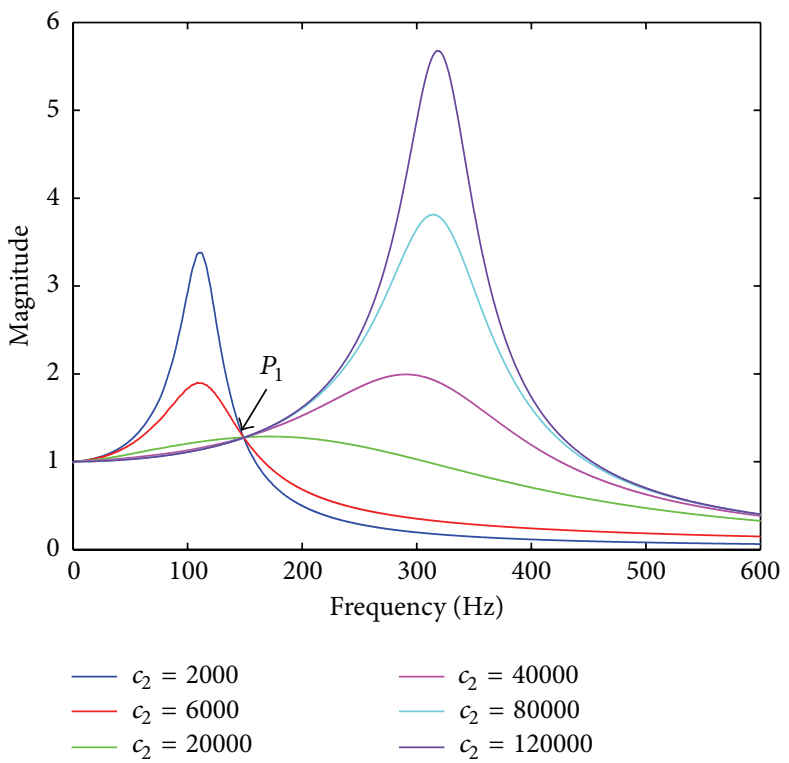

(c)

FIGURE 24: Force transmissibility curves of CM under $100 \mathrm{~N} \mathrm{FE}$ cases. (a) $n_{1}=1.5$; (b) $n_{1}=1$; (c) $n_{1}=0.85$.

the excitation amplitude is different. However, these nonlinear effects of damping exponent and excitation amplitude on the resonant peak and fundamental frequency can be taken into account if the $n_{1} \neq 1$ nonlinear models are used.

If $n_{1}=1$, based on the equivalence of mechanical impedance, a three-parameter model as illustrated in Figure 17 can be obtained from the Maxwell model, and the equivalent parameters are given by

$$
k_{A}=\frac{k_{1} k_{2}+k_{1} k_{3}+k_{2} k_{3}}{k_{2}+k_{3}}
$$

$$
\begin{aligned}
& k_{B}=\frac{k_{2}^{2} k_{4}}{\left(k_{2}+k_{3}\right)\left(k_{2}+k_{3}+k_{4}\right)}, \\
& c_{A}=\frac{k_{2}^{2} c}{\left(k_{2}+k_{3}\right)^{2}} .
\end{aligned}
$$

Thus the corresponding numerical values are $k_{A}=$ $6.100 \times 10^{6} \mathrm{~N} / \mathrm{m}$ and $k_{B}=8.866 \times 10^{6} \mathrm{~N} / \mathrm{m}$, and the critical damping coefficient $c_{c}$ is

$$
c_{c}=2 M \omega_{0}=2 \sqrt{k_{A} M}=27055.5 \mathrm{~N} / \mathrm{m} .
$$



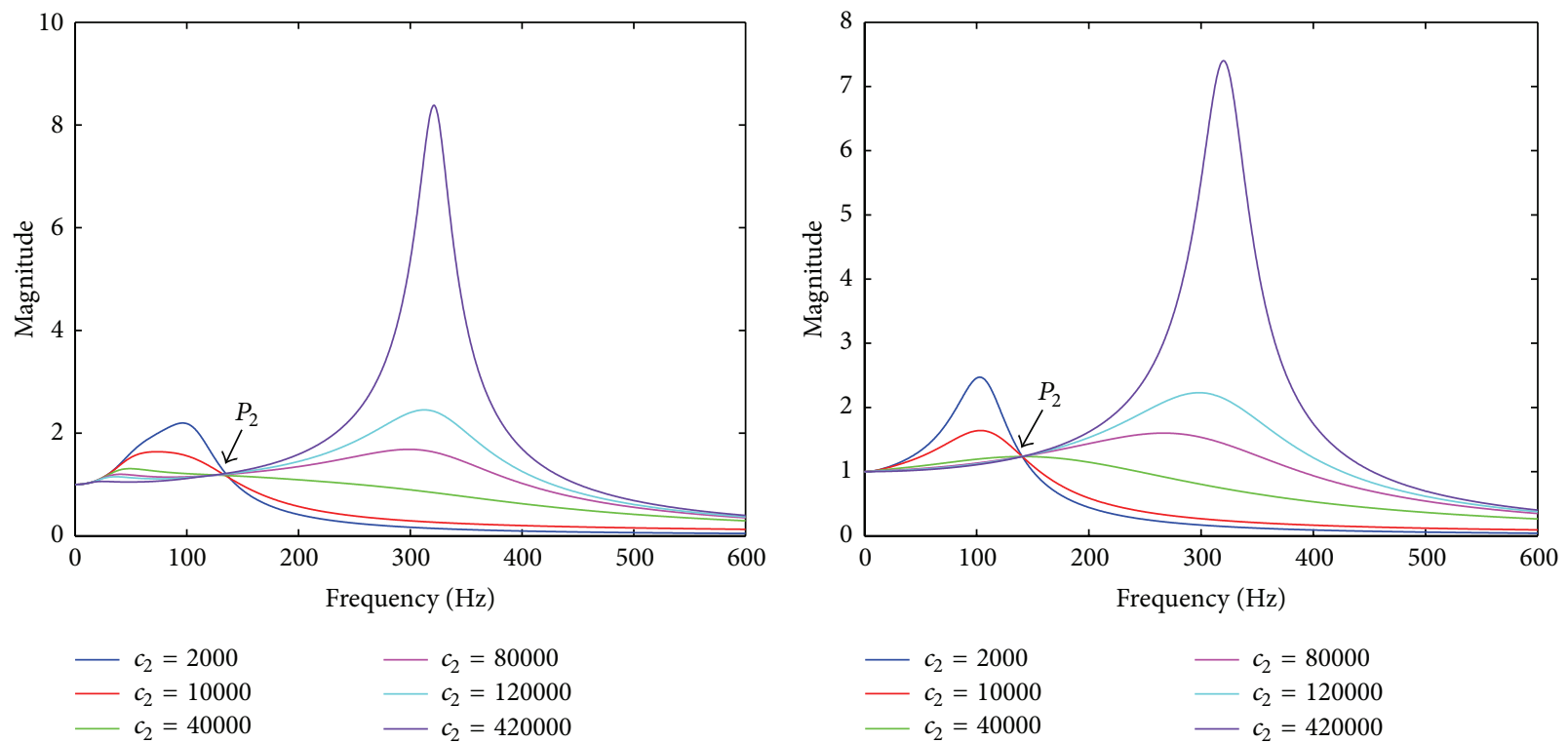

$\begin{aligned}-c_{2} & =80000 \\ c_{2} & =120000 \\ c_{2} & =420000\end{aligned}$

(a)

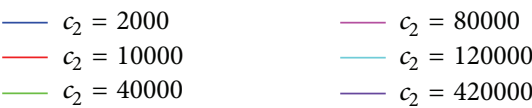

(b)

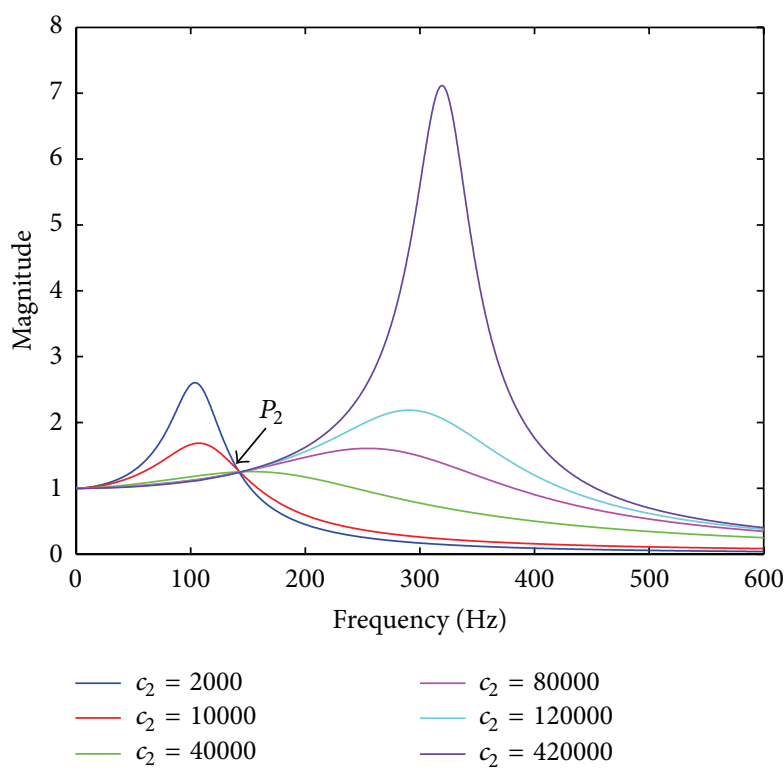

(c)

Figure 25: Absolute displacement transmissibility curves of CM under $0.76 \mathrm{~mm}$ FDE cases. (a) $n_{1}=1.5$; (b) $n_{1}=1$; (c) $n_{1}=0.85$.

Further, the optimal damping ratio is given by

$$
\varepsilon_{\mathrm{op}}^{A}=\frac{c_{A}}{c_{c}}=\frac{N}{4(N+1)} \sqrt{2(N+2)},
$$

where $N=k_{B} / k_{A}$. Accordingly, the optimal damping coefficient $c_{A}$ is equal to $10524.600 \mathrm{~N} \cdot \mathrm{s} / \mathrm{m}$, and the corresponding optimal damping ratio $\varepsilon_{\mathrm{op}}^{A}$ is 0.389 , so the transmissibility curve passes through the point $P_{1}$ or $P_{2}$ and has the lowest resonant peak at this time. Besides, if $c_{A} \rightarrow 0$, the fundamental frequency of this system $\omega_{1} \rightarrow \sqrt{k_{A} / M}$, and if $c_{A} \rightarrow \infty$, the fundamental frequency $\omega_{2} \rightarrow \sqrt{\left(k_{A}+k_{B}\right) / M}$.
4.2. Complicated Model. Letting $n=n_{1}=n_{2}$, Figures $18,19,20,21$, and 22 show the effects of flow index $n_{1}$ and damping coefficient $c_{1}$ on the performance of CM in different excitation cases.

As shown in Figures 18, 19, 20, 21, and 22, there are two resonant regions in the transmissibility curves. In the cases of $5.07 \mathrm{~mm}$ FDE, the differences of these three CMs become small, and the values of $f_{v}$ of $n_{1}=1.5 \mathrm{CM}$ are larger than those of others, so the damping exponent has significant effects on the performance of vibration isolation in FDE cases. For other excitation cases, the system variations are similar 


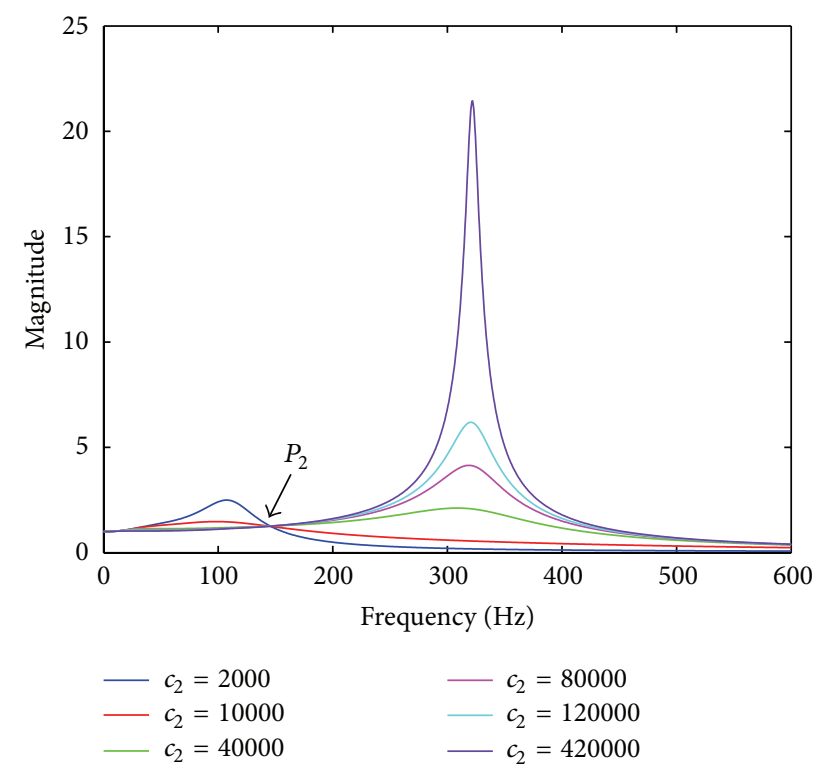

(a)

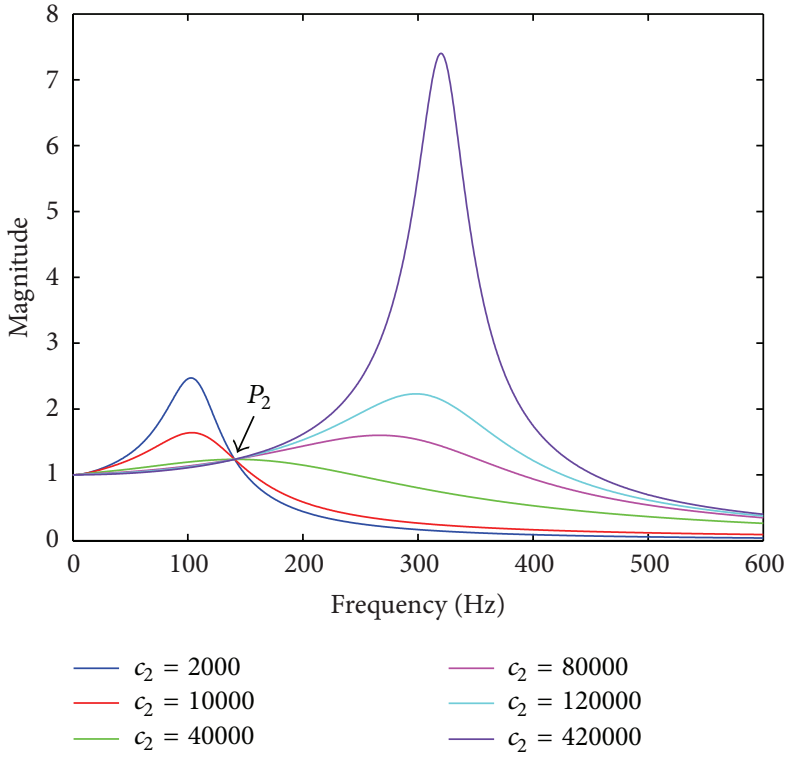

(b)

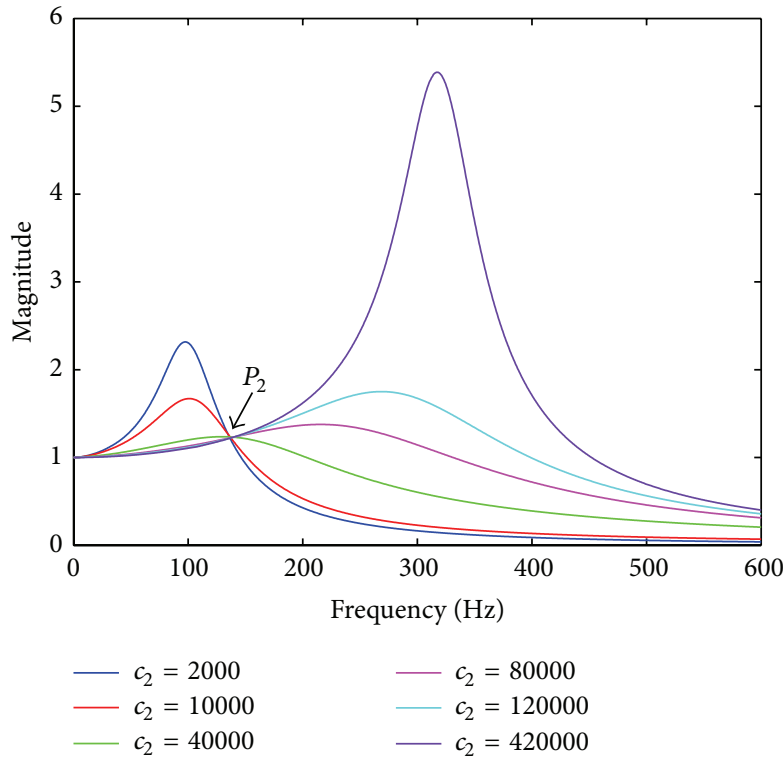

(c)

FIGURE 26: Absolute displacement transmissibility curves of CM under $5.07 \mathrm{~mm}$ FDE cases. (a) $n_{1}=1.5$; (b) $n_{1}=1$; (c) $n_{1}=0.85$.

to those of the GNM in Section 4.1 and will not be repeated again.

Letting $n=n_{1}=n_{2}$, Figures 23, 24, 25, 26, and 27 show the effects of flow index $n_{1}$ and damping coefficient $c_{2}$ on the performance of CM in different excitation cases.

As illustrated in Figures 23, 24, 25, and 26, two resonant regions are included in the transmissibility curves except the $n_{1}=1.5 \mathrm{FE}$ cases, and all of the transmissibility curves pass through one common point $P_{1}$ or $P_{2}$, which has the lowest resonant peak and it can serve as the critical point between the first resonant region and the second resonant region. If $c_{2} \rightarrow \infty$, the fundamental frequency of system increases into the second resonant region because of the rigid effect of damping coefficient $c_{2}$, while the resonant peak firstly reduces to $P_{1}$ or $P_{2}$ point and then increases with the raise of damping coefficient $c_{2}$. Furthermore, in the first resonant region, the smaller the damping coefficient $c_{2}$ is, the larger the high frequency roll-off rate is, but there is an opposite situation in the second resonant region. As indicated in Figure 27, in the cases of FE and $n_{1}=1.5$, since the damping coefficient $c_{2}$ is in parallel with the stiffness coefficient $k_{3}$, thus the resonant peak reduces and the fundamental frequency of system slightly decreases with the raise of damping coefficient $c_{2}$, which is similar to a single DOF system. However, the $n_{1}=1 \mathrm{CM}$ and the $n_{1}=0.85 \mathrm{CM}$ have high values of $f_{v}$; thus the fundamental frequency is easy to transfer from the first 


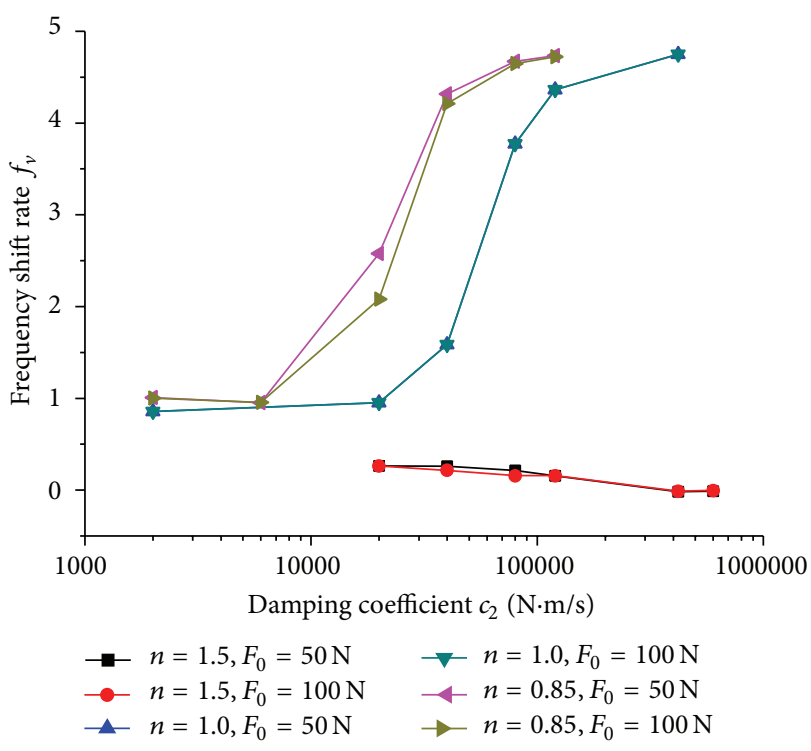

(a)

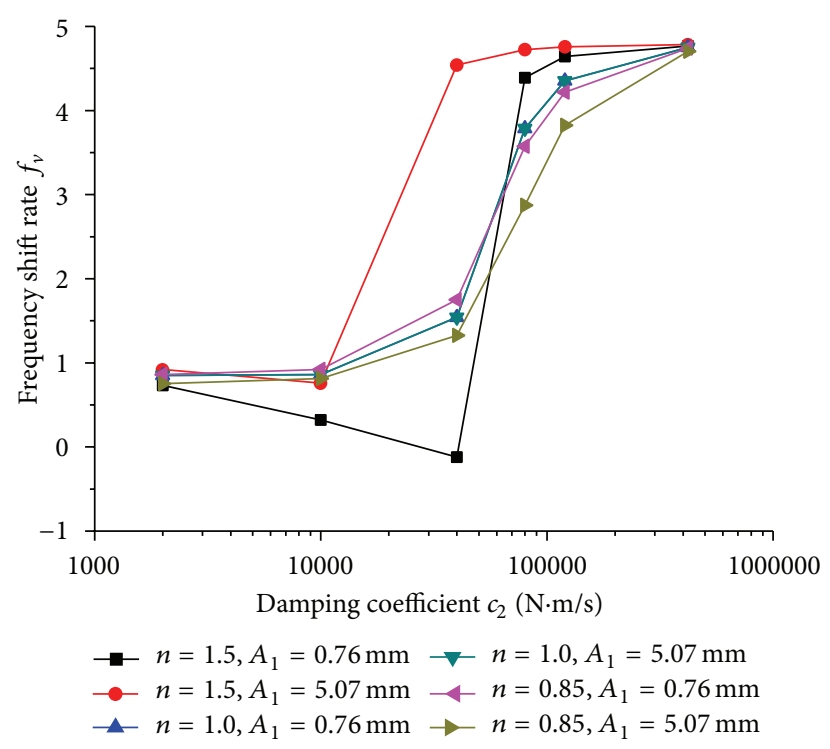

(b)

FIGURE 27: Frequency shift rates of CM. (a) FE cases; (b) FDE cases.

resonant region to the second one. In the cases of FDE and $n_{1}=1.5$, the fundamental frequency firstly reduces and then increases into the second resonant region, and it varies more fast than that of others with the increase of damping coefficient $c_{2}$. Thus the damping exponent and excitation amplitude have significant effects on the performance of vibration isolation, and only the $n_{1} \neq 1$ nonlinear models can consider these effects during the design of vibration isolators.

\section{Conclusion}

A non-Newton fluid microvibration isolator is studied in this paper, and it always behaves nonlinearly under the complicated effects of internal structure, external excitation, and fluid property, so this paper firstly presents several nonlinear models to characterize its vibration behaviors. On the basis of testing hysteretic loops, the GPS optimal algorithm of MATLAB optimization toolbox is used to identify the model parameters. With the use of the fourth-order RungeKutta method, the performance of these nonlinear models is further estimated. It can be seen that the simple model has the worst performance because of the large value of $\eta$. Due to the differences of deformation of fluid reservoir and the nonlinear effect of fluid, the GNM and the CM can properly characterize the physical vibration of isolator in the frequency band of 5-20 Hz. However, in the frequency band of 30-200 Hz, the Maxwell model performs better than others in the FE cases. As the microvibration isolator needs to experience the launch stage and the working state in orbit, during which the corresponding excitation amplitudes are significantly different, after the application of orthogonal testing method and the operation of parametric analysis with single variable method, the influences of several important factors, for example, damping coefficient and flow index, on the performance of vibration isolation, are obtained. The results show that only the GNM and CM can consider the above nonlinear effects in both the FE cases and the FDE cases, but the CM is not convenient to use in practice.

\section{Conflict of Interests}

The authors declare that there is no conflict of interests regarding the publication of this paper.

\section{Acknowledgments}

The authors gratefully acknowledge the financial support of Defense Basic Research Program through Grant nos. A2120110001 and B2120110011. This research work was also supported by the CAST Innovation Foundation of China under Grant no. CAST201208.

\section{References}

[1] W. H. Huang, D. Q. Cao, and Z. Y. Han, "Advances and trends in dynamics and control of spacecraft," Advances in Mechanics, vol. 42, no. 4, pp. 367-394, 2012 (Chinese).

[2] Z. H. Zhang, L. Yang, and S. W. Pang, "Jitter environment analysis for micro-precision spacecraft," Spacecraft Environment Engineering, vol. 26, no. 6, pp. 528-534, 2009 (Chinese).

[3] C. Blaurock, K. Liu, L. Dewell, and J. Alexander, "Passive isolator design for jitter reduction in the Terrestrial Planet Finder Coronagraph," in Optical Modeling and Performance Predictions II, vol. 5867 of Proceedings of SPIE, pp. 282-293, August 2005.

[4] J. C. Marr, "Space interferometry mission (SIM): overview and current status," in Interferometry in Space, vol. 4852 of Proceeding of SPIE, pp. 1-15, August 2002. 
[5] A. Rittweger, J. Albus, E. Hornung, H. Öry, and P. Mourey, "Passive damping devices for aerospace structures," Acta Astronautica, vol. 50, no. 10, pp. 597-608, 2002.

[6] X. J. Sun and J. R. Zhang, "Displacement transmissibility characteristics of harmonically base excited damper isolators with mixed viscous damping," Shock and Vibration, vol. 20, no. 5, pp. 921-931, 2013.

[7] P. Davis, D. Cunningham, and J. Harrell, "Advanced $1.5 \mathrm{~Hz}$ passive viscous isolation system," in Proceedings of the 35th AIAA/ASME/ASCE/AHS/ASC Structures, Structural Dynamics, and Materials Conference, pp. 2655-2665, Hilton Head, SC, USA, April 1994.

[8] E. Anderson, M. Trubert, J. Fanson, and P. Davis, “Testing and application of a viscous passive damper for use in precision truss structures," in Proceedings of the 32nd Structures, Structural Dynamics, and Materials Conference, pp. 2796-2808, AIAA Paper.

[9] R. A. Ibrahim, "Recent advances in nonlinear passive vibration isolators," Journal of Sound and Vibration, vol. 314, no. 3-5, pp. 371-452, 2008.

[10] Z. Q. Lang, X. J. Jing, S. A. Billings, G. R. Tomlinson, and Z. K. Peng, "Theoretical study of the effects of nonlinear viscous damping on vibration isolation of sdof systems," Journal of Sound and Vibration, vol. 323, no. 1-2, pp. 352-365, 2009.

[11] Z. K. Peng, Z. Q. Lang, L. Zhao, S. A. Billings, G. R. Tomlinson, and P. F. Guo, "The force transmissibility of MDOF structures with a non-linear viscous damping device," International Journal of Non-Linear Mechanics, vol. 46, no. 10, pp. 1305-1314, 2011.

[12] B. Tang and M. J. Brennan, "A comparison of two nonlinear damping mechanisms in a vibration isolator," Journal of Sound and Vibration, vol. 332, no. 3, pp. 510-520, 2013.

[13] Y. Ping, "Experimental and mathematical evaluation of dynamic behaviour of an oil-air coupling shock absorber," Mechanical Systems and Signal Processing, vol. 17, no. 6, pp. 1367-1379, 2003.

[14] Y. Ping, T. Yonghong, Y. Jianmin, and S. Nin, "Measurement, simulation on dynamic characteristics of a wire gauze-fluid damping shock absorber," Mechanical Systems and Signal Processing, vol. 20, no. 3, pp. 745-756, 2006.

[15] N. C. Shekhar, H. Hatwal, and A. K. Mallik, "Performance of non-linear isolators and absorbers to shock excitations," Journal of Sound and Vibration, vol. 227, no. 2, pp. 293-307, 1999.

[16] N. C. Shekhar, H. Hatwal, and A. K. Mallik, "Response of non-linear dissipative shock isolators," Journal of Sound and Vibration, vol. 214, no. 4, pp. 589-603, 1998.

[17] D. I. Narkhede and R. Sinha, "Behavior of nonlinear fluid viscous dampers for control of shock vibrations," Journal of Sound and Vibration, vol. 333, no. 1, pp. 80-98, 2014.

[18] L. Lu, G. Lin, and M. Shih, "An experimental study on a generalized Maxwell model for nonlinear viscoelastic dampers used in seismic isolation," Engineering Structures, vol. 34, pp. 111-123, 2012.

[19] L. Lu, C. C. Lin, and G. Lin, "Experimental evaluation of supplemental viscous damping for a sliding isolation system under pulse-like base excitations," Journal of Sound and Vibration, vol. 332, no. 8, pp. 1982-1999, 2013.

[20] J. Yang, Y. P. Xiong, and J. T. Xing, "Dynamics and power flow behaviour of a nonlinear vibration isolation system with a negative stiffness mechanism," Journal of Sound and Vibration, vol. 332, no. 1, pp. 167-183, 2013.

[21] Z. K. Peng, G. Meng, Z. Q. Lang, W. M. Zhang, and F. L. Chu, "Study of the effects of cubic nonlinear damping on vibration isolations using Harmonic Balance Method," International Journal of Non-Linear Mechanics, vol. 47, no. 10, pp. 1073-1080, 2012.

[22] B. Ravindra and A. K. Mallik, "Performance of non-linear vibration isolators under harmonic excitation," Journal of Sound and Vibration, vol. 170, no. 3, pp. 325-337, 1994.

[23] H. Laalej, Z. Q. Lang, S. Daley, I. Zazas, S. A. Billings, and G. R. Tomlinson, "Application of non-linear damping to vibration isolation: an experimental study," Nonlinear Dynamics, vol. 69, no. 1-2, pp. 409-421, 2012.

[24] J. Wang, S. G. Zhao, and D. F. Wu, "A test method of dynamic parameters of vibration isolators" (Chinese), Journal of Vibration Engineering. 

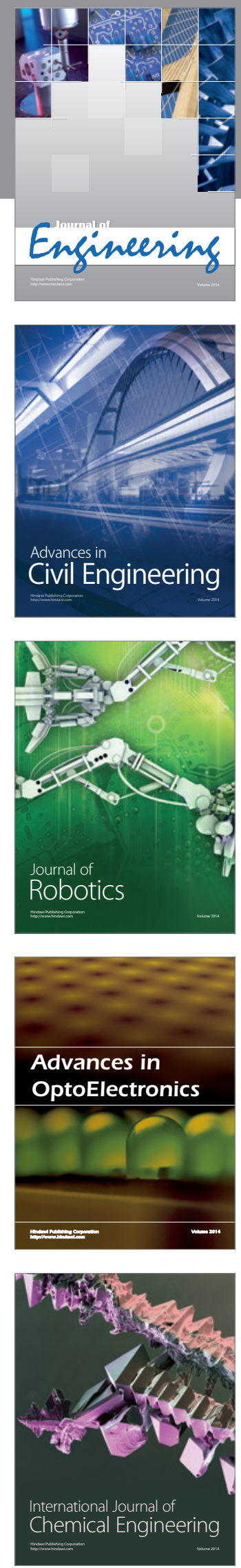

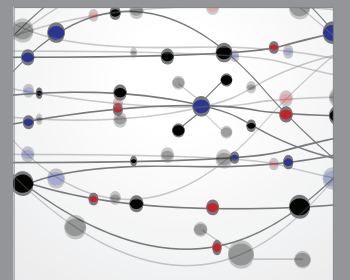

The Scientific World Journal
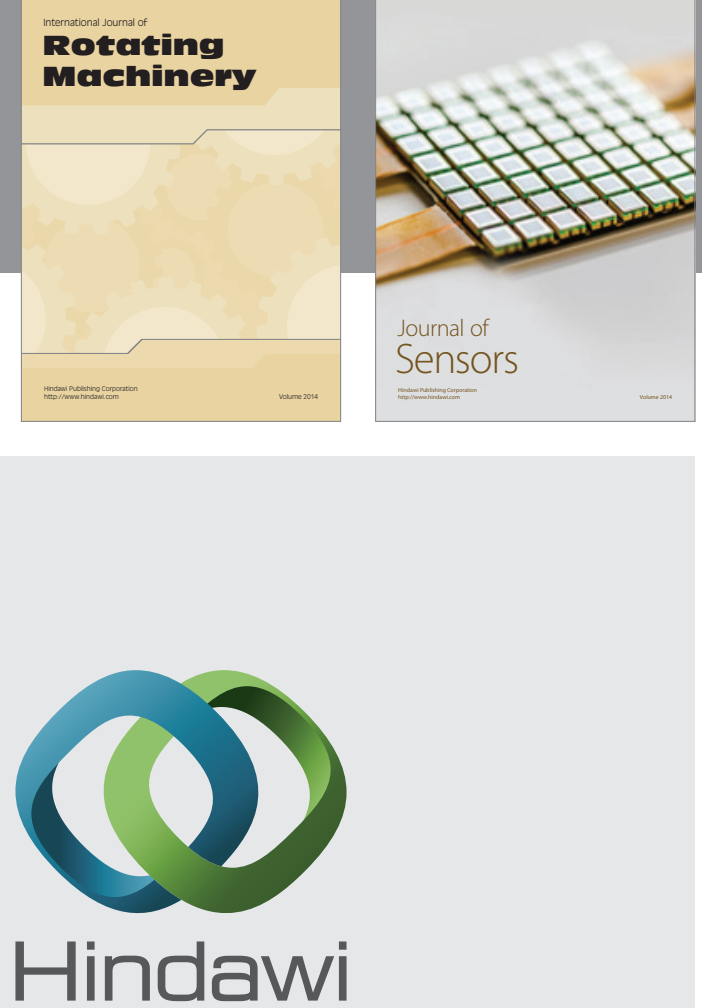

Submit your manuscripts at http://www.hindawi.com
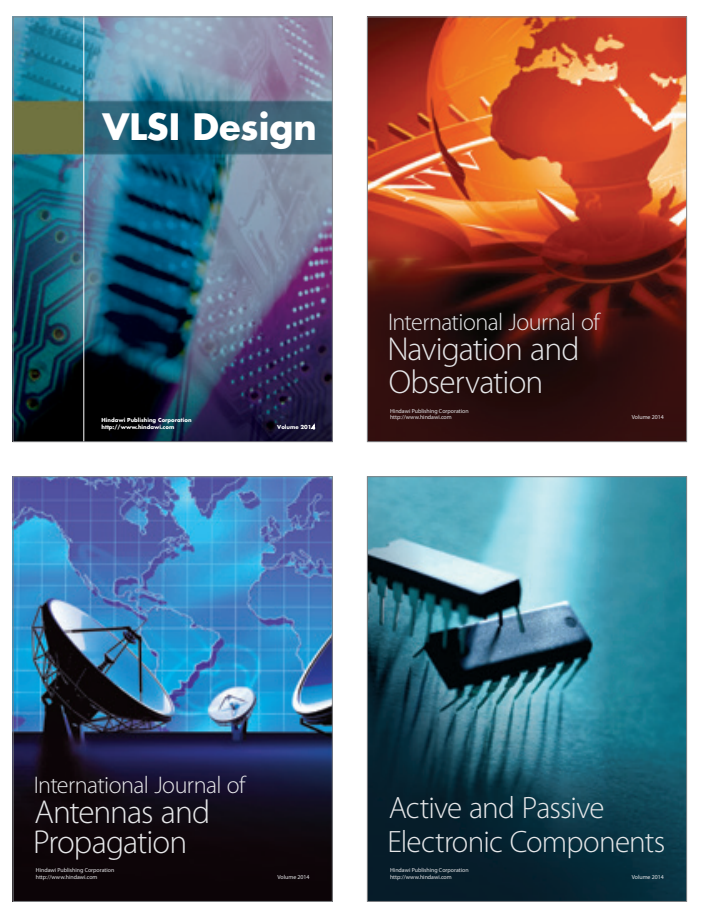
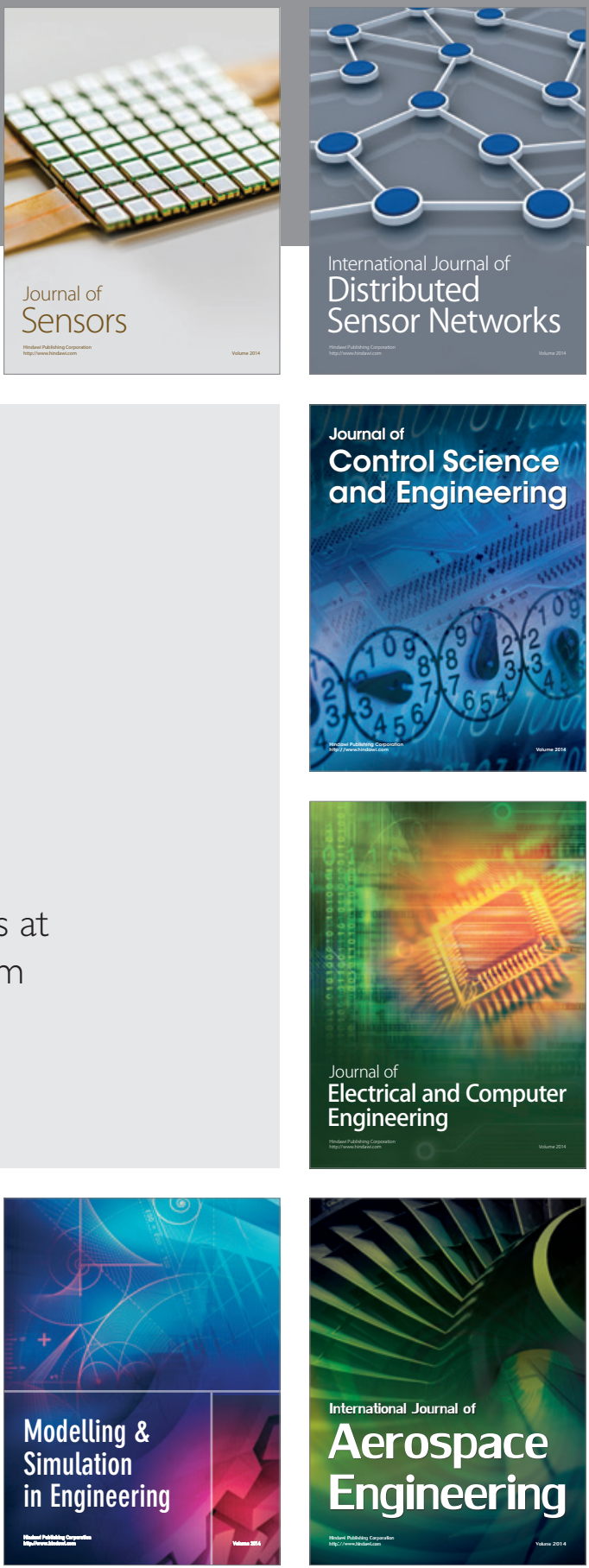

Journal of

Control Science

and Engineering
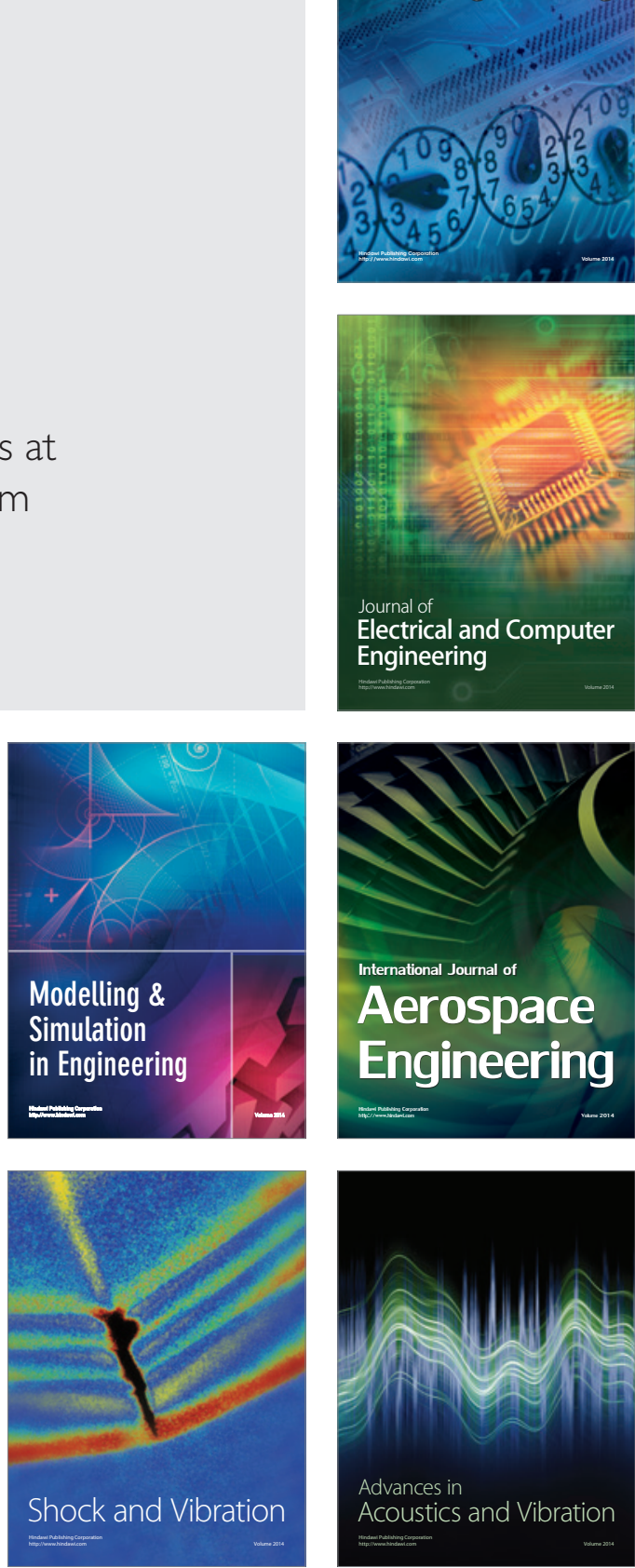